\title{
Mask-triggered thrust reversal in the negative compatibility effect
}

\author{
Thomas Schmidt $^{1}$ - Valerie Hauch ${ }^{2}$ - Filipp Schmidt ${ }^{2}$
}

Published online: 13 May 2015

(C) The Psychonomic Society, Inc. 2015

\begin{abstract}
Rapid motor responses to visual stimuli can involve both the activation and inhibition of motor responses. Here, we trace the early processing dynamics of response generation, examining whether activation and inhibition events form a strict sequence when elicited by sequential stimuli, as we would expect if motor events are driven by fast, stimulustriggered feedforward sweeps. We employed identical stimuli in two complementary paradigms. In response priming, responses to a target stimulus are speeded or slowed by a masked prime triggering the same or an alternative response, respectively. By prolonging the prime-target interval, the response-priming effect can reverse to form the negative compatibility effect $(N C E)$, especially when the mask contains response-relevant features. We report two experiments in which primed pointing movements going in ten possible directions were measured with response-relevant, response-irrelevant, or no masks interleaved between the primes and targets, while selective visual attention was varied. We showed that in response priming, initial responses are controlled exclusively by the prime. In the NCE, however, even the earliest movement phase is controlled jointly by the prime, mask, and target information, and a massive force in counterdirection to the primed response reverses the priming effects specifically for slow responses. We conclude that response priming reflects a strict sequence of feedforward response activations, whereas the activation/inhibition events in the NCE are not strictly serial, but integrate information from the different stimuli over time. Even though the mask features
\end{abstract}

Thomas Schmidt

thomas.schmidt@sowi.uni-kl.de

1 Faculty of Social Sciences, Experimental Psychology Unit, University of Kaiserslautern, Erwin-Schrödinger-Str. Geb. 57, 67633 Kaiserslautern, Germany

2 Justus-Liebig-University Giessen, Giessen, Germany and visual attention modulate the NCE, its major source is a mask-induced, direction-specific thrust reversal of the initial response.

Keywords Negative compatibility · Response priming · Response inhibition $\cdot$ Feature updating $\cdot$ Selective attention

Our visuomotor system has adapted to respond quickly to environmental stimuli, translating visual information into motor action almost in real time (Milner \& Goodale, 1995). Such visually guided action may involve both the activation and inhibition of motor responses. Consider a tennis player trying to return a ball. A skilled player will be able to use the ball's early trajectory to prepare a good response. But when the ball touches the net and unexpectedly changes its trajectory, the current response must be quickly inhibited and replaced with a new one, possibly in the middle of a sprint in the wrong direction.

To understand how visual stimuli can exert online control over motor activation and inhibition, it is necessary to figure out fundamental properties of their processing dynamics. One such property is whether neuronal processing is feedforward or recurrent. Lamme and Roelfsema (2000; Bullier, 2001; Kirchner \& Thorpe, 2006; Thorpe, Fize, \& Marlot, 1996; VanRullen \& Koch, 2003; VanRullen \& Thorpe, 2001, 2002) have proposed that a newly appearing visual stimulus elicits a wave of neuronal activation that spreads from posterior to anterior areas of the cortex quickly enough that the cells first reached by the wave front have to pass on their activation essentially with the next spike fired, without being able to process feedback about their own activity. In macaque monkeys, this fast feedforward sweep can reach executive motor areas within about $150 \mathrm{~ms}$ after stimulus onset, and it could be the basis of stimulus-driven motor responses that are fast and automatized and may not be accessible to conscious awareness. While the feedforward wave front is progressing, loops of recurrent feedback quickly form in the wake of the wave 
front. Finally, the recurrent loops forming between widespread brain areas establish a necessary condition for visual awareness and cognitive control (Lamme \& Roelfsema, 2000).

\section{Motor activation in response priming}

The time course of stimulus-triggered motor activation can be studied in response-priming tasks (Klotz \& Neumann, 1999; Klotz \& Wolff, 1995; Vorberg, Mattler, Heinecke, Schmidt, \& Schwarzbach, 2003). In a typical response-priming task, participants perform a speeded response to a target stimulus that is preceded by a prime stimulus triggering either the same response as the target (consistent prime) or the opposite response (inconsistent prime). In many experiments, the target serves the additional purpose of reducing the visibility of the prime by backward masking (Breitmeyer \& Öğmen, 2006), but primes can just as well be shown unmasked in a flanker design (Eriksen \& Eriksen, 1974; F. Schmidt, Haberkamp, \& Schmidt, 2011; Schwarz \& Mecklinger, 1995). Consistent primes speed up responses to the target, whereas inconsistent primes slow down responses, and this response-priming effect increases with increasing time interval between the prime onset and the target onset (stimulus-onset asynchrony, SOA; Vorberg et al., 2003).

Response priming mainly occurs because of a motor conflict generated by the prime- and target-triggered motor activations. This was first demonstrated in the time course of lateralized readiness potentials ( $L R P S)$, which start out timelocked to the prime, first develop in the direction specified by the prime, and only later proceed in the direction specified by the actual target (Eimer \& Schlaghecken, 1998; Klotz, Heumann, Ansorge, \& Neumann, 2007; Leuthold \& Kopp, 1998; Vath \& Schmidt, 2007; Verleger, Jaśkowski, Aydemir, van der Lubbe, \& Groen, 2004). The same pattern of sequential response activation can be observed in overt response behavior, such as the kinematics of primed pointing responses (Brenner \& Smeets, 2004; T. Schmidt, 2002) or the time course of force deployment in isometric buttonpresses (F. Schmidt, Weber, \& Schmidt, 2014). These studies have shown that inconsistent primes are able to mislead pointing movements in the wrong direction, such that the initial behavioral response is time-locked to the prime, first proceeds in the direction specified by the prime, and only then proceeds in the target direction (F. Schmidt et al., 2014; T. Schmidt, 2002; T. Schmidt, Niehaus, \& Nagel, 2006; T. Schmidt \& Schmidt, 2009; cf. Song \& Nakayama, 2009). The priming effect increases with prime-target SOA, because the prime has progressively more time to direct the response in the correct or the incorrect direction. Also, an inconsistent prime can provoke a response error, so that incorrect responses are fast errors that occur predominantly in inconsistent trials at long SOAs. Vorberg et al. (2003), Mattler and Palmer (2012), and
Schubert, Palazova, and Hutt (2013) have provided models and simulations of these response-activation processes.

\section{Reversal of priming effects}

Surprisingly, the response-priming effect can reverse at long prime-target SOAs. This negative compatibility effect (NCE), discovered by Eimer and Schlaghecken (1998), has been used in many experiments to study the time course of response inhibition (for reviews, see Klauer \& Dittrich, 2010; Sumner, 2007). Eimer and Schlaghecken (1998) employed double arrows $(<<$ or $>>)$ as the primes and targets. A 17-ms prime was immediately followed by a 100 -ms mask constructed from the superposition of left- and right-pointing double arrows. The mask was immediately followed by a target that was either consistent with the prime (pointing in the same direction, thus requiring the same response) or inconsistent (requiring the opposite response). Surprisingly, the authors found that responses were slower and error rates were higher when the primes and targets were consistent than when they were inconsistent-a reversal of the priming effect normally expected. They traced the effect in lateralized readiness potentials, observing a sequence of three response activations: an initial activation of the prime-related response at a fixed time after prime onset, followed by a transient activation of the opposite (antiprime) response, and finally an activation of the targetrelated response. Obviously, the antiprime activation was what reversed the priming effect. Lingnau and Vorberg (2005) examined the dynamics of this effect in a parametric study employing a prime arrow, a metacontrast mask $70 \mathrm{~ms}$ after prime onset, and a target arrow. They showed that priming effects turn from positive to negative at progressively longer prime-target SOAs, and that stimuli that are stronger in terms of retinal size or proximity to fixation evoke a larger NCE that begins at earlier SOAs.

Eimer and Schlaghecken (1998) explained reverse priming effects by an automatic inhibition ${ }^{1}$ of the prime response and concomitant disinhibition of the antiprime response in a winner-takes-all system. Their 1998 article suggested that this inhibition is triggered by the mask because the mask interrupts the sensory evidence for a prime-related response. In subsequent studies, they argued that this "removal" of sensory

\footnotetext{
${ }^{1}$ In principle, inhibitory control of motor actions in priming tasks can occur in a bottom-up or a top-down fashion (Boy, Husain, \& Sumner, 2010), and consequently can take the form of reactive or proactive control (Braver, 2012). Feedforward processing could be the basis of stimulusdriven inhibition, such as is required when processing a sudden stop signal (de Jong, Coles, Logan, \& Gratton, 1990; Logan \& Cowan, 1984; van Gaal, Ridderinkhof, van den Wildenberg, \& Lamme, 2009) or when alternative responses inhibit each other in a winner-takes-all system. In contrast, recurrent processing could be the basis of top-down cognitive control (Allport, Styles, \& Hsieh, 1994; Rogers \& Monsell, 1995).
} 
evidence coincides with the prime's disappearance from visual awareness (Eimer \& Schlaghecken, 2002; Schlaghecken \& Eimer, 2002), and that the prime itself triggers its own inhibition, provided that its initial "perceptual strength" exceeds a threshold (Eimer \& Schlaghecken, 2002, 2003; also see Klapp \& Hinkley, 2002). Praamstra and Seiss (2005) suggested that the prime could also trigger its own inhibition automatically, independent of its conscious visibility. Indeed, an NCE can be obtained, no matter whether the prime is strongly masked (Verleger et al., 2004) or clearly visible (Jaśkowski, 2007b).

In contrast to the self-inhibition accounts, many authors have pointed out the role of the mask features in the inhibition process. Eimer and Schlaghecken (1998) already noted that following the prime with a mask that superimposes a prime and an antiprime is tantamount to presenting the prime first and then the antiprime on top of it. This involvement of features that are already associated with specific motor responses renders the mask response-relevant, in contrast to a responseirrelevant mask that contained only neutral features. Lleras and Enns (2004) proposed that the NCE results entirely from perceptual object updating, which simply replaces the initial representation of the prime features with a representation of the antiprime features, with a concomitant reversal of the motor activation process. They considered this to be "based on a rather ordinary mechanism of perceptual updating rather than on the inhibition of unconsciously activated responses" ( $p$. 490), but this updating would ultimately lead to a sequence of motor activations, first by the prime and then by the antiprime features in the mask. Verleger et al. (2004) proposed a similar account through the concept of an "active mask" that directly addresses the motor system if it contains responserelevant features. In contrast, Jaśkowski and PrzekorackaKrawczyk (2005; Jaśkowski, 2007a) viewed response inhibition as a genuine process on top of feature-based priming. They proposed that any mask acts as an emergency brake that leads to mask-triggered inhibition of the currently active response to the prime, irrespective of the mask's ability to reduce the prime's visibility. Jaśkowski (2007a) pointed out that mask-triggered inhibition may be stronger following relevant than following irrelevant masks, but in contrast to the objectupdating hypothesis, inhibition need not be limited to response-relevant masks. Several studies have now systematically compared different mask types and generally found that the reversal in priming effects is more likely to occur when the mask is response-relevant (e.g., Jaśkowski \& Ślósarek, 2007; Lleras \& Enns, 2004). However, NCEs have also been observed with response-irrelevant masks (e.g., Lleras \& Enns, 2004; Schlaghecken \& Eimer, 2006; Sumner, 2008).

All these accounts of the NCE have one common feature: They imply that (1) the initial response should be in the prime direction and time-locked to prime onset, and (2) the later response in the antiprime direction should be time-locked to the mask. Thus, all of the theories agree that motor events in the NCE, be they excitatory or inhibitory, are triggered in sequence by stimulus events. It is this notion that we examine in the present article.

\section{Rapid-chase theory: Identifying strictly sequential processing}

Lamme and Roelfsema's (2000) theory of feedforward versus recurrent processing can explain response priming as resulting from sequential feedforward sweeps triggered in turn by the primes and targets. However, the notion of a feedforward processing stage in human vision is debatable, because feedback mechanisms within and between visual areas can be rapid (Bullier, 2001, 2004; Roland, 2010; Sillito, Cudeiro, \& Jones, 2006), and information might be processed at different rates in parallel streams (Chen et al., 2007; Merigan \& Maunsell, 1993). Because it is mostly impossible to directly observe the feedforward sweep in human participants, we have provided some bridging principles that link feedforward processing to overt behavior in response priming and related tasks.

Indeed, several studies have shown that the early time course of primed pointing movements (T. Schmidt et al., 2006; T. Schmidt \& Schmidt, 2009) and LRPs (Vath \& Schmidt, 2007) depends only on prime, but not on the target characteristics. In other words, the initial response is controlled exclusively by the prime, as would be predicted for a simple feedforward system that processes primes and targets in strict sequence. T. Schmidt et al. (2006) studied primed pointing responses to color stimuli, using primes and targets of either high or low color saturation as well as different types of targets. The initial time courses of the priming effects depended exclusively on the onset time and saturation of the primes but were independent of the onset time of the actual targets. Similarly, Vath and Schmidt (2007) studied keypress responses with primes and targets that could independently be high or low in color saturation. Again, the initial priming effects in LRPs depended only on the prime onset and prime saturation, but were independent of target onset or target saturation. In both studies, only later segments of the priming effects were influenced by the characteristics of the actual target, but the initial time course of the response was invariant when locked to prime onset. This pattern of strictly sequential response control by primes and targets can also be demonstrated in the time course of response force in isometric keypress responses (F. Schmidt et al., 2014) and in analyses of response time distributions (F. Schmidt \& Schmidt, 2014; see Panis \& Hermens, 2014, for an alternative technique).

T. Schmidt et al. (2006; T. Schmidt et al., 2011) proposed that primes and targets elicit feedforward sweeps that traverse the visuomotor system in strict sequence, without any temporal overlap (rapid-chase theory of response priming). The prime and target sweeps are able to directly initiate the motor 
responses assigned to them without a need for conscious control (the principle of direct parameter specification: Neumann, 1990; cf. Kunde, Kiesel, \& Hoffmann, 2003). The prime signal reaches motor areas first, initiating a response and continuing to direct the response until the target signal takes over response control from the prime. Because the response delay is much longer than the prime-target interval, the overt response starts after both primes and targets have been presented, but initially it follows information from the prime, not the target. Priming effects in response times and error rates increase with prime-target SOA because the prime has progressively more time to drive the response on its own when the target is further delayed. The feedforward properties of such a system would show in the time course of the motor response as follows (chase criteria: T. Schmidt et al., 2006; see T. Schmidt, 2014, for formal definitions):

1. Prime rather than target signals determine the onset and initial direction of the response (initiation criterion).

2. Target signals are able to influence the response before it is completed (takeover criterion).

3. The movement kinematics initially depend on the prime characteristics only and are independent of all target characteristics (independence criterion).

The initiation and independence criteria describe exclusive initial response control by the prime, and the takeover criterion is needed to guarantee that the target has not been ignored. The chase criteria bridge the gap between a theoretically proposed feedforward sweep and the observable behavior, such that a stimulus-response system meeting these criteria will be behaviorally equivalent to a simple feedforward system. Note that the rapid-chase account does allow for local recurrence, as long as sequential input signals still lead to strictly sequential motor output. Further note that rapid-chase processing requires that the first sweep be independent of the second sweep, but not vice versa. Therefore, the criteria demand that initial processing be controlled exclusively by the prime, but not that later processing be controlled exclusively by the target ( $\mathrm{T}$. Schmidt, 2014).

In this article, we used the chase criteria to test a fundamental question implicit in all major theories of response priming and the NCE: Can effects be explained by a strict sequence of excitatory or inhibitory motor events triggered in turn by the prime, mask, and target stimuli? We trace response priming and the NCE in the time course of unfolding pointing responses, looking for motor effects that are time-locked to the onsets of the various stimuli. We showed that such timelocking is evident in the regular, positive response-priming effect, whereas the time course of the NCE defies an explanation in terms of strictly sequential response activation or inhibition. We concluded that regular response priming can indeed be explained by a simple sequence of visuomotor feedforward sweeps. In contrast, the NCE involves the temporal integration of prime and target information prior to movement onset, revealing a fundamental processing difference between the positive and reverse priming effects. Strikingly, our data reveal that the response inhibition in the NCE is in fact a massive counterforce to the primed response, starting $350 \mathrm{~ms}$ after mask onset and working precisely contrary to the prime direction.

\section{Experiment 1}

Experiment 1 was designed to investigate the NCE in primed pointing movements. The experiment had several objectives. First, we were interested in how the NCE would develop over time in the unfolding motor response. Second, we tried to clarify the role of the mask in the NCE by comparing trials without any masks to trials with response-irrelevant and response-relevant masks. Third, we wanted to elucidate the role of visual attention in the NCE. Previous research had indicated that response-priming effects are boosted by visual attention, both space-based (T. Schmidt \& Seydell, 2008) and feature-based (F. Schmidt \& Schmidt, 2010). The role of attention in the NCE is less clear. Sumner, Tsai, Yu, and Nachev (2006) showed that the NCE is larger when the prime location is attended rather than unattended, and that the effect is similar to when the prime's luminance contrast is increased. However, it is unclear whether attention affects the NCE by enhancing response-relevant features or by boosting response inhibition. One additional aim of the experiment was therefore to get further insight into how attention amplifies the NCE.

We employed a paradigm introduced by T. Schmidt and Seydell (2008). We studied the impact of endogenously controlled attention (Yantis \& Serences, 2003) on response priming by presenting a circular configuration of ten possible targets (horizontal and vertical bars) preceded by ten primes (also horizontal and vertical bars) and ten masks at the same positions (Fig. 1). The stimulus configurations were such that neighboring stimuli would be alternately horizontal and vertical, and a horizontal target or prime would always lie opposite to a vertical one. Participants responded to one pair of opposing targets by pointing to the horizontal one of the pair (twoalternative forced choice). To know which of the target pairs to respond to, they had to process a cue presented before prime onset, which indicated the relevant pair of opposing targets (the relevant locations). We expected that visual attention would gradually mold into the cued stimulus positions, allowing for more efficient stimulus processing at the attended locations (T. Schmidt \& Seydell, 2008). We varied the time available for the deployment of attention by using two different cueing intervals, 100 and $500 \mathrm{~ms}$, based on our previous experiments. 


$\begin{array}{lllll}\text { Fixation } & \text { Cue } & \text { Primes } & \text { Masks } & \text { Targets } \\ \text { point } & 50 \mathrm{msec} & \text { (con, incon) } & \text { (no, irrel., rel.) } & \text { (until response) } \\ & & 17 \mathrm{msec} & 17 \mathrm{msec} & \end{array}$

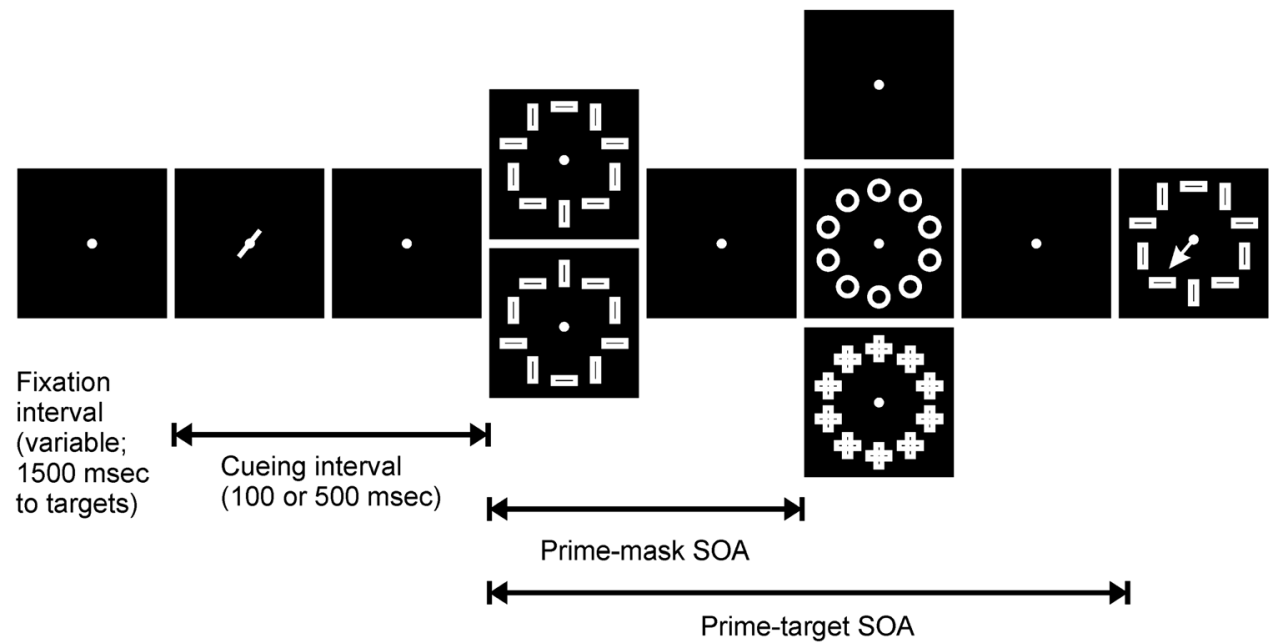

Fig. 1 Paradigm used in all experiments. In every trial, ten primes were presented, followed by ten targets, such that the stimuli were alternately horizontal and vertical, and each vertical stimulus lay opposite a horizontal one. The cue could point along any of the five possible axes and marked the response-relevant target locations. Participants had to

This paradigm has two major advantages over traditional cueing tasks. First, visual selection of the cued locations is necessary for performing the task in the first place ("selection for action"; Allport, 1989). Therefore, the cue cannot be ignored, as in tasks of the Posner (1980) type. Second, in the absence of spatial selection, no spatially directed influence of the primes on the pointing movements would be expected, because numerous primes of opposite orientations are arranged evenly around the resting position. Therefore, any observation of spatially directed priming effects would demonstrate that spatial selection must have taken place, constituting a check whether the attentional manipulation was effective.

Finally, the most important purpose of the experiment was to apply the chase criteria to the NCE. To work out specific predictions from rapid-chase theory, consider a spatial measure of the priming effect, defined as the difference between finger positions in consistent and inconsistent trials at corresponding points in time (Fig. 2a). The spatial priming effect is expected to start out in the negative direction if an inconsistently primed response lags behind a consistently primed response (regular, positive priming effect). In a feedforward system (Fig. 2b), priming effects should start at a fixed time after prime onset and follow an invariant initial time course; that is, there is a stretch of time over which the movement is exclusively controlled by the prime for a period depending on the prime-target SOA. As the target takes control of the response, the priming trajectories branch off from the initial common time course in the order of SOAs. In contrast, in a system in which the prime, mask, and target signals are integrated, fused, or have temporal overlap prior to movement point from the central fixation point to the horizontal target on the cued axis. Between the prime and target onsets, either no masks, responseirrelevant masks (circles), or response-relevant masks (horizontal and vertical bars superimposed) were presented. The two experiments only differed in the timing of these stimuli

onset (Fig. 2c), the onset of priming effects should still be time-locked to the prime onset. However, they should form a branching pattern right from the start of the movement, because progressively shorter SOAs would lead to a progressively larger influence of the target information relative to the prime information. ${ }^{2}$

\section{Method}

Participants Eight students from the University of Giessen (age 21-31 years, four of them male, all of them right-handed) volunteered for a payment of $€ 42$. Their vision was normal or corrected to normal, and all of them gave informed consent.

Apparatus and stimuli The experiment was controlled by a $300-\mathrm{MHz}$ PC driving a 14-in. VGA color monitor $(640 \times$ 480 pixels) in synchrony with the monitor retrace rate of $60 \mathrm{~Hz}$. The monitor image was projected onto a workspace via a set of two mirrors, such that participants had the impression that the stimuli appeared directly on the workspace, where participants could interact with them. The workspace was tilted toward the participant by $44^{\circ}$ out of the transversal plane at $70 \mathrm{~cm}$ viewing distance, and was illuminated such that participants could view their hand superimposed on the stimuli. Pointing responses were recorded by a POLHEMUS

\footnotetext{
${ }^{2}$ Grainger, Scharnowski, Schmidt, and Herzog (2013) distinguished feature fusion from feature integration. Integration describes any process whereby two stimuli have a combined impact instead of separate impacts, whereas fusion describes a special case in which the stimuli are combined into a single conscious representation.
} 

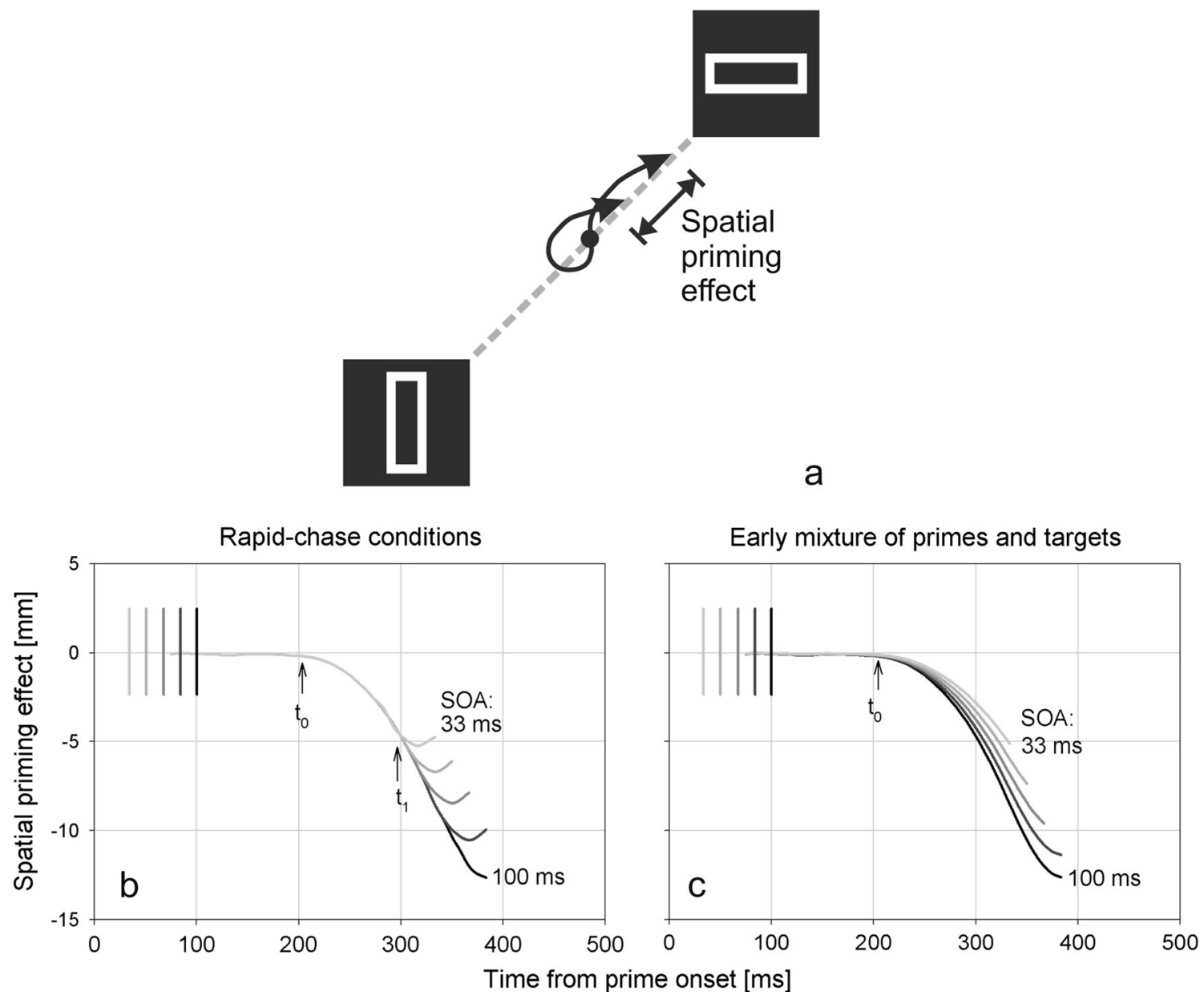

Fig. 2 (a) Spatial priming effects are defined as the spatial difference of the sensor positions in inconsistent minus consistent trials when the movement trajectories are projected onto the imaginary line connecting the cued positions. Negative values indicate that the sensor position is closer to the target in consistent trials than in inconsistent trials at the same point in time. (b) Spatial priming effects expected under rapid-chase conditions. When response control is strictly sequential, the initial time course of the pointing response must be invariant when time-locked to prime onset, because the response must at first be determined exclusively by the prime. Spatial priming effects for progressively later prime-target SOAs should branch off one by one from the initial invariant time course. (c) Spatial priming effects expected when the prime and target information integrates before movement onset. The shorter the prime-target stimulus-onset asynchrony, the smaller the impact of the prime on the motor response. Therefore, spatial priming effects are expected to form a fan-shaped pattern right from the start of the overt response
FASTRAK magnetic tracking device in synchrony with stimulus presentation, and were sampled at $120 \mathrm{~Hz}$. Participants used a sensor stylus to point to stimuli projected onto the workspace.

The primes and targets were small outline rectangles $\left(1.40^{\circ}\right.$ $\times 0.47^{\circ} ; 1 \mathrm{~mm} \approx 0.082^{\circ}$ of visual angle). The masks, if presented, consisted either of outline circles (diameter $1.13^{\circ}$ ) or of horizontal and vertical rectangles (superimposed to form a "plus" sign). The fixation point in the center of the screen had a diameter of $0.12^{\circ}$. The ten prime, mask, and target stimuli were spaced equally around the fixation point at regular $36^{\circ}$ intervals, forming a circle with radius $4.68^{\circ}$. These stimuli were preceded by a spatial cue at the fixation point, consisting of two bars $\left(0.94^{\circ}\right.$ each $)$ pointing at two opposite target locations. All stimuli were presented in white $\left(83.00 \mathrm{~cd} / \mathrm{m}^{2}\right)$ against a dark background $\left(0.07 \mathrm{~cd} / \mathrm{m}^{2}\right)$.
Procedure Each trial started with the appearance of the central fixation point (see Fig. 1). The participants placed the pointing stylus on the fixation point and started the trial by pressing the space bar. After a variable delay, the cue was presented for $50 \mathrm{~ms}$ in the center of the screen. Following a cue-prime SOA of either 100 or $500 \mathrm{~ms}$, the ten primes were presented for $17 \mathrm{~ms}$. Then, $50 \mathrm{~ms}$ after prime onset, masks were presented for $17 \mathrm{~ms}$ at the same positions as the primes, consisting of either no stimulus at all (no mask), neutral circles (irrelevant mask), or horizontal and vertical bars superimposed (relevant mask). Finally, the ten targets were presented at prime-target SOAs of either 83, 133, 183, or $233 \mathrm{~ms}$ (chosen to cover a range from strongly positive to clearly negative priming effects) and remained on screen until participants had finished the response. Participants responded by performing a speeded pointing movement with the tip of 
the stylus (held in the preferred hand like a pencil) from the fixation point to the horizontal target at one of the two cued locations. The time interval from fixation onset to target onset was constant at $1,500 \mathrm{~ms}$, to allow for optimal preparation for response to the target. The prime shapes were either all consistent or all inconsistent with the target shapes. All stimulus combinations of consistency, cueing interval, prime-target SOA, and mask type occurred equiprobably and pseudorandomly in a completely crossed repeated measures design (16 repetitions of each combination per participant and session, allowing us to obtain reliable data patterns at the level of individual participants). In each trial, the correct target location was drawn randomly from a uniform distribution.

We employed two tasks under identical stimulus conditions: a speeded target identification task (Target ID, designed to measure priming effects) and a prime identification task (Prime ID, designed to measure discrimination performance for the primes). In the Target ID task, participants pointed as quickly as possible to the horizontal one of the two cued target stimuli, as described above. In the Prime ID task, they tried to point without time pressure to the horizontal one of the two cued prime stimuli. Note that both tasks were two-alternative forced choice tasks (Green \& Swets, 1966; Macmillan \& Creelman, 2005), because participants had to consider the two cued locations only, which always contained one horizontal and one vertical prime and target. Furthermore, both tasks employed the same stimuli and cueing procedure, and we took care that the Prime ID task would focus on exactly the stimulus information (prime shape) that would drive the priming effect in the Target ID task (T. Schmidt \& Vorberg, 2006).

Data acquisition stopped when the sensor had entered a sphere of $17-\mathrm{mm}$ radius around any of the two admissible target positions for at least $50 \mathrm{~ms}$. The arrival time was defined as the time when the sensor first crossed the boundary of this sphere. After each response, a 100-ms, 1000-Hz tone indicated that the response had been registered. Error feedback was not given.

Participants performed five 1-h sessions of Target ID, followed by a 1-h session of Prime ID, each consisting of one practice block followed by 16 blocks of 48 trials. After each block, participants received summary feedback about their average response time and were allowed to take a brief pause. After the final session, participants were debriefed and received an explanation of the experiment.

Statistical methods Trials in Target ID task were excluded if arrival times were shorter than $100 \mathrm{~ms}$ or longer than $1,000 \mathrm{~ms}$ ( $1.30 \%$ of trials). In addition, trials were excluded when participants failed to respond or when they hit one of the invalid target locations (5.79\% of trials in Target ID, $2.61 \%$ in Prime ID). Only correct responses entered the analysis of movement trajectories. The error rates were arcsine-transformed to fit the requirements for analysis of variance. All analyses for the
Prime ID task were calculated, depending on the experiment, with the factors Mask (M), Prime-Target SOA (S), and Cueing Interval (I). All analyses for the Target ID task were calculated separately for each masking condition (no mask, irrelevant mask, or relevant mask) and, depending on the experiment, with the factors Consistency (C), Prime-Target SOA, and Cueing Interval. All analyses of variance are reported with Huynh-Feldt corrected $p$ values; $F$ values are reported with subscripts indicating the respective effects. All significant effects are reported.

Pointing movements were sampled in all three spatial dimensions and then projected onto the line connecting the correct and incorrect target locations, yielding single-valued, time-dependent measures of the sensor positions along that line for consistent and inconsistent trials [movement trajectories: $\left.f_{\text {con }}(t), f_{\text {incon }}(t)\right]$. The trajectorial priming functions were defined as the differences between inconsistent and consistent movement trajectories, $f_{\text {incon }}(t)-f_{\text {con }}(t)$. Kinematic parameters were extracted from these functions by a jackknifing procedure and statistically tested with the corrections derived by Ulrich and Miller (2001). ${ }^{3}$ We extracted several parameters to characterize the priming process in both the temporal and spatial domains. Arrival times were calculated by setting a spatial criterion $(17.00-\mathrm{mm}$ radius around correct target) and then evaluating the point in time when the movement crossed this boundary. Arrival times are given relative to target onset. Temporal priming effects were defined as the differences between the arrival times $a_{i}$ in inconsistent and consistent trials $\left(a_{\text {incon }}-a_{\text {con }}\right)$. Spatial priming effects were defined on the basis of the trajectorial priming function, which describes how far the pointing movements in inconsistent trials lag behind those in consistent trials. The peak priming amplitude was defined as the maximum negative value (in millimeters) achieved by the trajectorial priming function, or, where appropriate, as the maximum positive value. We also extracted the time of this peak amplitude.

\section{Results}

Overall time course of pointing responses Figure 3 shows the time courses of pointing responses separately for all masking conditions and cueing intervals. In consistent trials, responses start roughly $300 \mathrm{~ms}$ after prime onset and then proceed in the correct direction, whereas responses in inconsistent trials tend to be delayed (a regular, positive responsepriming effect). The priming effect is especially strong in the absence of masks, where it grows with prime-target SOA. It is very small for response-irrelevant masks, and it actually

\footnotetext{
$\overline{3}$ Averaged trajectories entering the jackknifing procedure were smoothed with a T4253H filter (Velleman, 1980). The filter had little effect on the averaged trajectories but smoothed their time derivative, which was helpful in extracting the peak amplitude parameters.
} 
reverses for response-relevant masks: At long SOAs, pointing movements in consistent trials start to lag behind those in inconsistent trials (an NCE). Note that responses at progressively longer SOAs also take longer to be initiated and carried out. Further note that favorable attentional conditions enhance all aspects of the movement kinematics: At the 500-ms cueing interval, which should allow for near-optimal attentional preparation, responses start earlier, proceed faster, and are completed sooner than at the 100-ms cueing interval (F. Schmidt \& Schmidt, 2010; T. Schmidt \& Seydell, 2008). In the following analysis, we look at the temporal and spatial markers of these effects in more detail, considering each mask type separately.

Target ID: No masks In the absence of masks, only positive priming effects were obtained. Arrivals at the correct target position occurred later in inconsistent than in consistent trials $\left[F_{\mathrm{C}}(1,7)=64.22, p<.001\right.$, calculated relative to target onset $]$. Similarly, more response errors (arrivals at the position opposite the correct target) occurred in inconsistent than in consistent trials $\left[F_{\mathrm{C}}(1,7)=7.67, p=.028\right]$ (Fig. 4, left panels). Error rates increased with increasing prime-target $\mathrm{SOA}\left[F_{\mathrm{S}}(3,21)=\right.$ $6.47, p=.017]$. Priming effects in arrival times as well as error rates increased with prime-target $\mathrm{SOA}\left[F_{\mathrm{C} \times \mathrm{S}}(3,21)=4.01, p\right.$ $\left.=.028 ; F_{\mathrm{C} \times \mathrm{S}}(3,21)=6.89, p=.018\right]$ as well as with cueing interval $\left[F_{\mathrm{C} \times \mathrm{I}}(1,7)=20.70, p=.003 ; F_{\mathrm{C} \times \mathrm{I}}(1,7)=11.67, p=\right.$ .011]. Arrivals occurred progressively earlier with longer prime-target $\operatorname{SOAs}\left[F_{\mathrm{S}}(3,21)=35.18, p<.001\right]$ and occurred earlier for the longer cueing interval $\left[F_{\mathrm{I}}(1,7)=44.40, p<\right.$ $.001]$.

If the initial pointing response was controlled entirely by the prime, the trajectorial priming functions should initially be invariant and lie exactly on top of each other. The actual priming functions (Fig. 5, left panels) show no sign of such an invariance in the initial time course of spatial priming effects: The functions fan up immediately after response onset, following the pattern shown in Fig. $2 \mathrm{c}$ rather than the one predicted for a rapid-chase system in Fig. 2b. The peak amplitude of the trajectorial priming effect was strongly negative [analysis of intercept: $\left.F_{\text {int }}(1,7)=28.43, p=.001\right]$ and was larger for the longer cueing interval $\left[F_{\mathrm{I}}(1,7)=55.81, p<.001\right]$. In the shorter cueing interval, it increased with prime-target SOA $\left[F_{\mathrm{S} \times \mathrm{I}}(3,21)=4.39, p=.037\right]$. The peak amplitude was reached earlier with a longer cueing interval $\left[F_{\mathrm{I}}(1,7)=7.14, p\right.$ $=.032]$ and a shorter prime-target $\operatorname{SOA}\left[F_{\mathrm{S}}(3,21)=141.41, p\right.$ $<.001]$.

Target ID: Irrelevant masks Priming effects were still obtained when response-irrelevant masks followed the primes, but they were much smaller than in the no-mask condition. Arrivals occurred later in inconsistent than in consistent trials $\left[F_{\mathrm{C}}(1,7)=10.93, p=.013\right]$, and inconsistent trials also produced more response errors $\left[F_{\mathrm{C}}(1,7)=9.38, p=.018\right]$ (Fig. 4, middle panels). Error rates increased with increasing prime- target SOA $\left[F_{\mathrm{S}}(3,21)=12.58, p<.001\right]$. Priming effects increased with prime-target SOA only in error rates $\left[F_{\mathrm{C} \times \mathrm{S}}(3\right.$, $21)=6.20, p=.003]$, whereas priming effects in arrival times were affected by neither prime-target SOA nor cueing interval. Arrivals occurred earlier with longer prime-target SOAs $\left[F_{\mathrm{S}}(3,21)=31.48, p<.001\right]$ and the longer cueing interval $\left[F_{\mathrm{I}}(1,7)=68.60, p<.001\right]$.

Peak amplitudes of the spatial priming effects were also strongly reduced when compared to the no-mask condition, but they were still detectable $\left[F_{\text {int }}(1,7)=11.37, p=.012\right]$. Interestingly, they tended to decrease with prime-target SOA at the longer cueing interval $\left[F_{\mathrm{S} \times \mathrm{I}}(3,21)=3.37, p=.053\right]$ (Fig. 5, middle panels). The peak amplitude was reached earlier with shorter prime-target SOAs $\left[F_{\mathrm{S}}(3,21)=50.93, p<\right.$ $.001]$ and by trend at the longer cueing interval $\left[F_{\mathrm{I}}(1,7)=\right.$ $5.08, p=.059]$. Thus, when the cueing interval was long, spatial priming effects showed signs of a reversal toward the NCE. This effect of prime-target SOA on the time of peak amplitude was more pronounced at the longer cueing interval $\left[F_{\mathrm{S} \times \mathrm{I}}(3,21)=4.78, p=.011\right]$. Importantly, we found no indication of an early invariance in the trajectorial priming functions, which fanned out immediately at the time of response onset.

Target ID: Relevant masks When primes were followed by response-relevant masks, we expected reversals of the priming effects. Indeed, only the shortest SOA led to regular, positive priming effects in which arrivals occurred later in inconsistent than in consistent trials. With longer prime-target SOAs, the priming effects reversed $\left[F_{\mathrm{C} \times \mathrm{S}}(3,21)=11.82, p<.001\right]$ (Figs. 3 and 4, right panels). Priming effects in arrival times were not modulated by cueing interval. Arrivals occurred earlier with longer prime-target $\operatorname{SOAs}\left[F_{\mathrm{S}}(3,21)=36.01, p<\right.$ $.001]$ and at the longer cueing interval $\left[F_{\mathrm{I}}(1,7)=25.48, p=\right.$ $.001]$. Error rates increased with increasing prime-target SOA $\left[F_{\mathrm{S}}(3,21)=5.08, p=.008\right]$ but displayed no significant priming effects.

To work out the influence of mask type on the sign of the priming effect in arrival times, we performed an additional analysis of variance with the factors Cueing Interval, Mask Type (M), and Prime-Target SOA, with priming effects as the dependent variable. Priming effects in arrival times were larger at the longer cueing interval $\left[F_{\mathrm{I}}(1,7)=11.35, p=.012\right]$ and strongly depended on mask type $\left[F_{\mathrm{M}}(2,14)=77.82, p<\right.$ $.001]$. We observed an interaction of cueing interval and mask type $\left[F_{\mathrm{I} \times \mathrm{M}}(2,14)=14.09, p=.007\right]$, reflecting the large cueing effect on priming specific to the no-mask condition. Importantly, there was also an interaction of mask type and SOA $\left[F_{\mathrm{M} \times \mathrm{S}}(6,42)=8.81, p=.021\right]$, confirming the different time courses of priming in the different masking conditions. Restricting the analysis to the conditions with masks present yielded a main effect of SOA $\left[F_{\mathrm{S}}(3,21)=6.07, p=.002\right]$, indicating that priming effects became smaller (more 

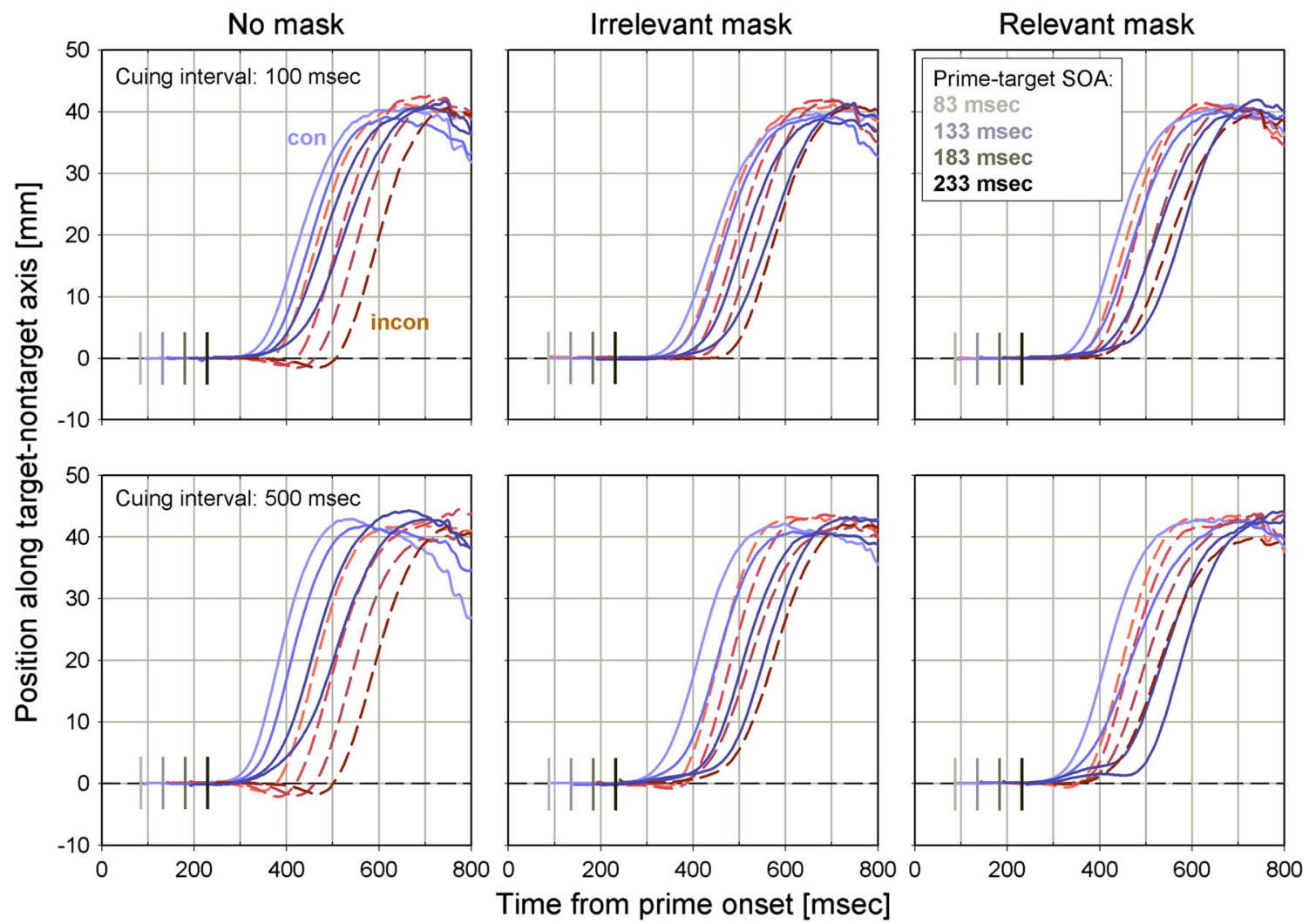

Fig. 3 Movement trajectories in Experiment 1, target identification task. The solid and dashed curves indicate consistent and inconsistent trials, respectively. All trajectories are locked to prime onset; the target onset is

marked by vertical bars. Positive values indicate movement toward the location of the correct target, and negative values, movement toward the location of the incorrect target

negative) with SOA. Importantly, a strong main effect of mask type emerged $\left[F_{\mathrm{M}}(1,7)=22.03, p=.002\right]$ and an interaction of mask type with SOA $\left[F_{\mathrm{M} \times \mathrm{S}}(3,21)=9.54, p=.002\right]$, reflecting the differences in the time courses of the priming effect for relevant and irrelevant masks. The main effect of mask type and the interaction of mask type and SOA were also significant in the error rates.

The gradual reversal of the priming effect with increasing prime-target SOA in the relevant-mask condition is beautifully reflected in the spatial priming effects (Fig. 5, right panels). Generally, these effects are bimodal, with an early negative peak and a second, positive peak. With increasing prime-target SOA, the first peak becomes weaker and earlier, whereas the second peak becomes stronger and earlier, until the second peak dominates the pattern at the expense of the first peak. We decided to analyze the two peaks separately. The amplitude of the first peak (going negative and indicating regular priming) $\left[F_{\text {int }}(1,7)=3.74, p=.035\right]$ decreased with prime-target SOA $\left[F_{\mathrm{S}}(3,21)=8.95, p=.012\right]$ but was not significantly modulated by cueing interval (Fig. 5, right panels). The peak amplitude was reached progressively earlier with increasing prime-target $\mathrm{SOA}\left[F_{\mathrm{S}}(3,21)=4.51, p=.014\right]$, and this effect was more pronounced at the longer cueing interval $\left[F_{\mathrm{S} \times \mathrm{I}}(3\right.$, 21 ) $=3.74, p=.027]$. The amplitude of the second peak (going positive and indicating reverse priming) $\left[F_{\text {int }}(1,7)=16.55, p\right.$
$=.005]$ also increased with prime-target $\mathrm{SOA}\left[F_{\mathrm{S}}(3,21)=\right.$ $5.38, p=.020]$, but was not significantly modulated by cueing interval. Even though the second peak seems to be reached progressively earlier for longer prime-target SOAs, the time of the positive peak was significantly modulated by neither prime-target SOA nor cueing interval. Note that there is no notable temporal invariance in any of these effects: Plotting the effects relative to target rather than to prime onset does not align the peaks, and none of them is time-locked to either prime, mask, or target onset.

Direction-specific counterforce production Ocampo and Finkbeiner (2013) analyzed pointing responses in the NCE paradigm as a function of movement onset time. They showed that the NCE only occurs in trials that start late, whereas in trials with fast response onsets only positive priming effects occur. Their analysis of onset times was possible because movements started on a trajectory perpendicular to the workspace, whereas ours started in the center of the workspace. In inconsistent trials, it was therefore difficult for us to determine whether a movement started before or after the motor conflict: Depending on the interplay of response activation and response inhibition, trajectories might veer toward the incorrect target, but they might also be stalled by inhibition before starting in the correct direction, rendering any onset 


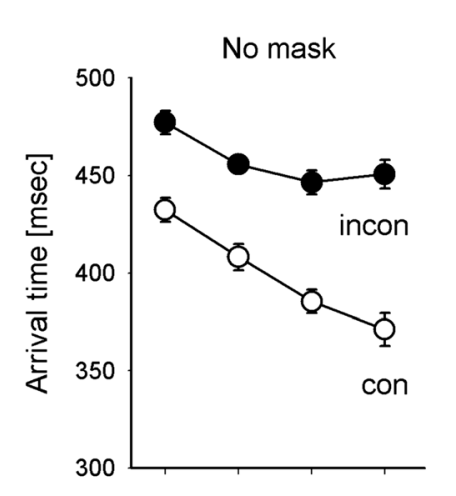

Cueing interval: $100 \mathrm{~ms}$
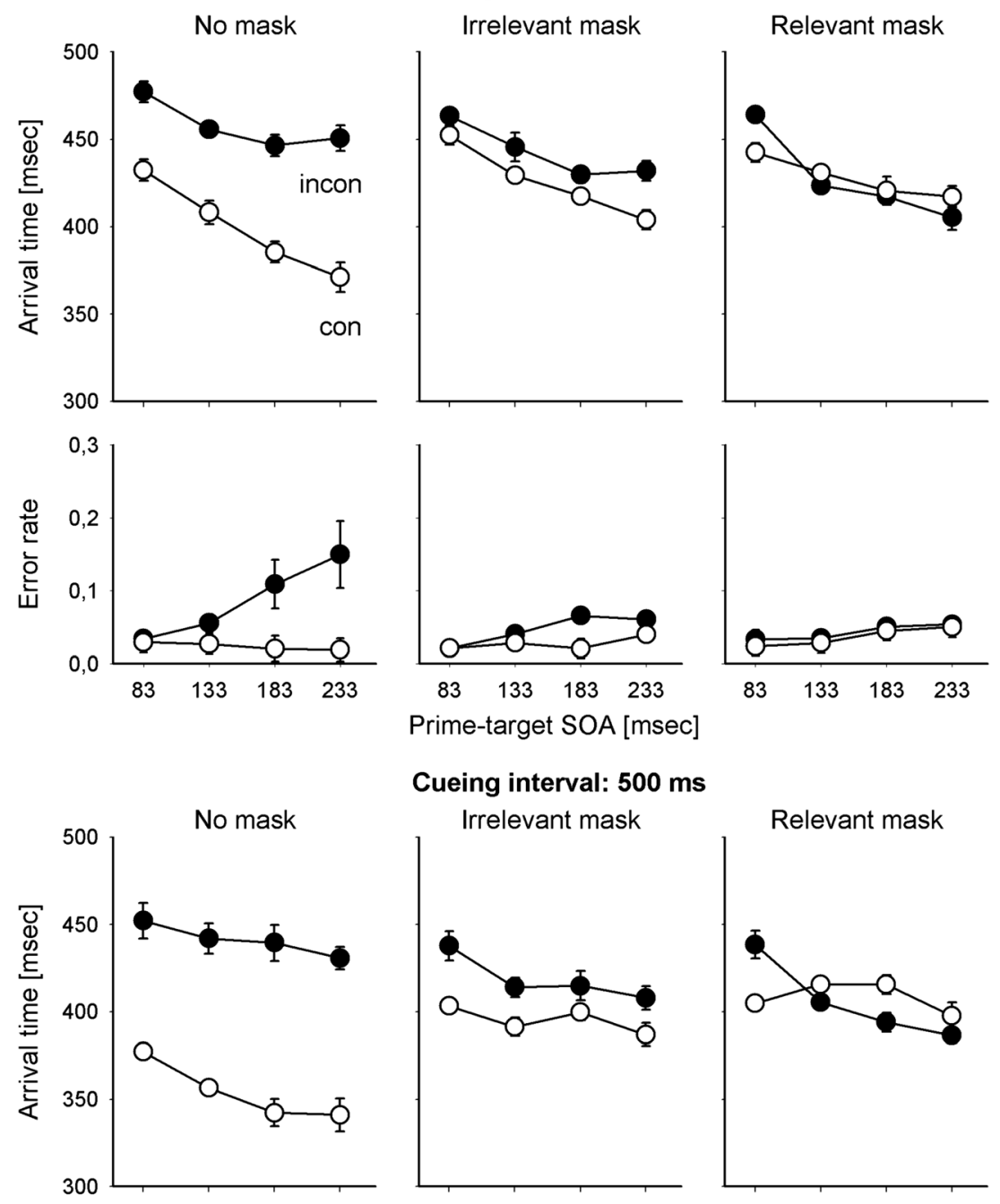

Cueing interval: $\mathbf{5 0 0} \mathbf{~ m s}$
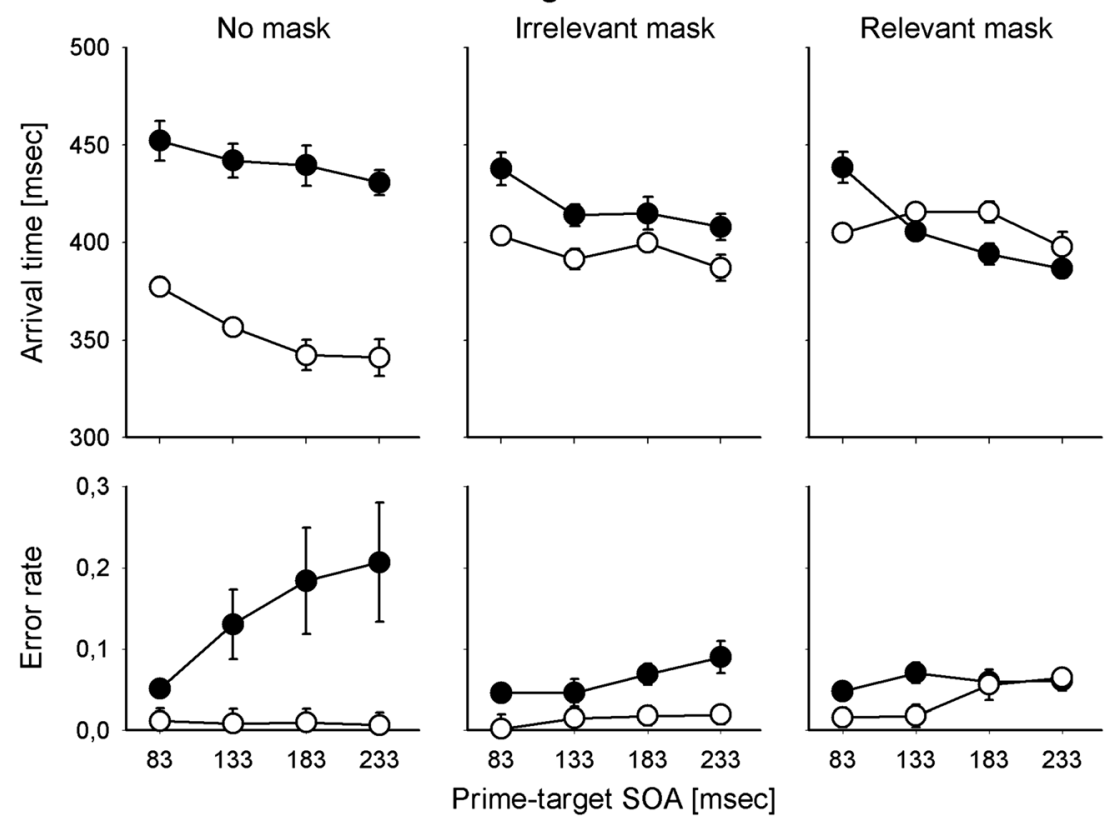

Fig. 4 Arrival times (upper panels) and error rates (lower panels) in Experiment 1, target identification task. Here and in subsequent figures, error bars denote the standard errors of the means with between-subjects variance removed, to account for the repeated measures design (Cousineau, 2005)

criterion unreliable. In consistent trials, however, onsets could be determined easily. ${ }^{4}$

Movement onset was defined as the time when the sensor had moved $10 \mathrm{~mm}$ away from fixation in the direction of the correct target (on the basis of individual trials, since an

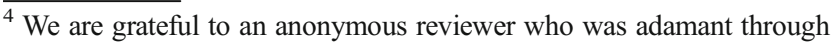
two review processes that we analyze the effect of onset time, even though we first believed it was impractical, given the geometry of our task. It turns out that this analysis was the key to understanding the NCE.
}

analysis by jackknifing was not possible here). The distribution of onset times was split into quartiles separately for each participant and condition. Figure 6 plots the results of this analysis (consistent trials only) and reveals a surprising effect. When responses start early (1st quartile), the pointing movements in consistent trials always proceed continuously to the correct target position. But for progressively later response onsets (2nd to 4 th quartiles), there is an increasing tendency for initial movement in the opposite direction. Since we are only looking at consistent trials, this movement is against the 

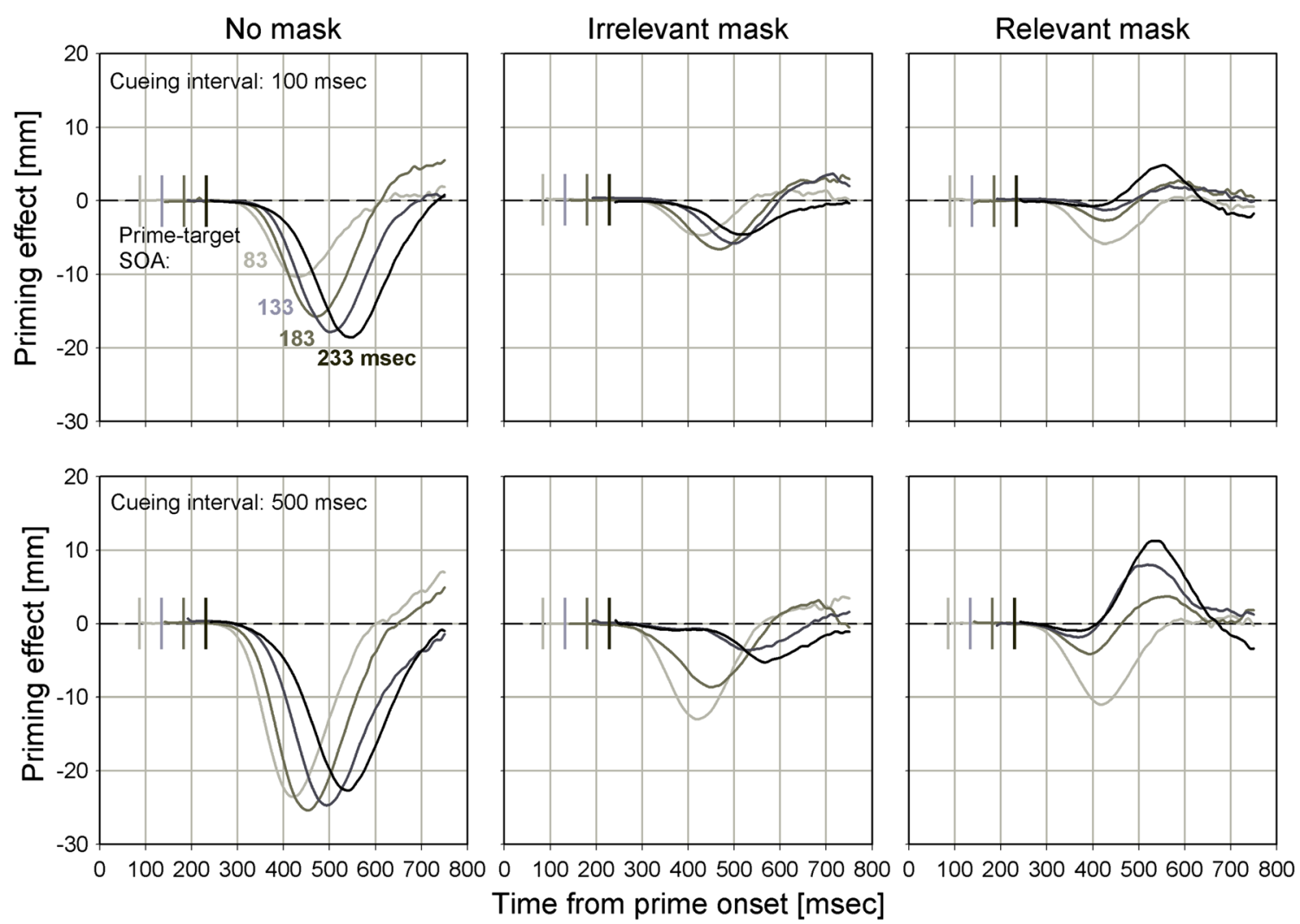

Fig. 5 Trajectorial priming functions in Experiment 1, target identification task, defined as the difference between the corresponding solid and dashed curves in Fig. 3. Negative values indicate that the movement in consistent trials leads the movement in inconsistent trials at the same point in time

direction specified by both the prime and the target. This effect is massive in size and increases with prime-target SOA. It starts about $350 \mathrm{~ms}$ after mask onset (400 ms after prime onset) and seems to be time-locked to one of those stimuli. Importantly, it occurs for response-irrelevant as well as relevant masks, indicating that it is not based on the specific mask features, and it is strongly reduced when masks are absent. Moreover, it is counteracted (or removed) by the target, as is indicated by its dependence on prime-target SOA. Finally, it is spatially directed, indicating a force in the precise antiprime direction.

We performed a statistical test on these inhibitory effects by calculating the time integral under the negative-going part of the trajectories in Fig. 6, using jackknifing techniques. An analysis of variance with the factors Cueing Interval, PrimeTarget SOA, and Onset Quartile $(\mathrm{Q})$ revealed that in the presence of response-relevant masks, the area below the $x$-axis (in $\mathrm{ms} \cdot \mathrm{mm})$ increases with onset quartile $\left[F_{\mathrm{Q}}(3,21)=21.70, p<\right.$ $.001]$ and prime-target $\mathrm{SOA}\left[F_{\mathrm{S}}(3,21)=6.33, p=.003\right]$, with a significant interaction of both factors indicating an overadditive effect $\left[F_{\mathrm{Q} \times \mathrm{S}}(9,63)=3.80, p=.001\right]$. The pattern was similar in the irrelevant-mask condition, in which the area increased with quartile $\left[F_{\mathrm{Q}}(3,21)=24.38, p<.001\right]$ and prime-target $\mathrm{SOA}\left[F_{\mathrm{S}}(3,21)=3.93, p=.023\right]$, with an interaction again indicating an overadditive effect $\left[F_{\mathrm{Q} \times \mathrm{S}}(9,63)=\right.$ $3.69, p=.001]$. When masks were absent, the area only increased with onset quartile $\left[F_{\mathrm{Q}}(3,21)=6.94, p=.002\right]$. Note that none of these analyses reveals any effect of cueing interval on this area measure of response inhibition. Significant intercept tests indicated significant negative-going responses in the no-mask as well as the irrelevant-mask and relevant-mask conditions $\left[F_{\text {int }} \mathrm{s}(1,7)=7.34,44.12\right.$, and 29.44; $p \mathrm{~s} \leq .030, .001$, and .001 , respectively]. However, the area measure has a small bias toward negative values because it truncates positive values that occur by random fluctuation, so the intercept effect in the no-mask condition should be treated with caution.

Prime ID The presence of masks strongly impaired discrimination of the prime (Fig. 7). At the 100-ms cueing interval, logistic regression with the factors Prime-Target SOA and Mask Type (standardized so that the intercept indicates overall performance relative to chance level) showed that prime identification performance was better without a mask than with either relevant or irrelevant masks, Wald's $\chi^{2}{ }_{M}(1)=145.76$, $p<.001$, and there was an interaction of mask type and SOA, $\chi_{\mathrm{M} \times \mathrm{S}}^{2}(1)=8.45, p=.004$. Separate analyses of the three masking functions showed that they were all significantly different from chance, all $\chi^{2}{ }_{\text {int }} \mathrm{s}(1) \geq 16.01$, all $p \mathrm{~s}<.001$, and that only for the no-mask condition did performance increase with prime-target SOA, $\chi^{2}{ }_{S}(1)=10.56, p=.001$. At the $500-\mathrm{ms}$ cueing interval, mask type similarly determined prime 


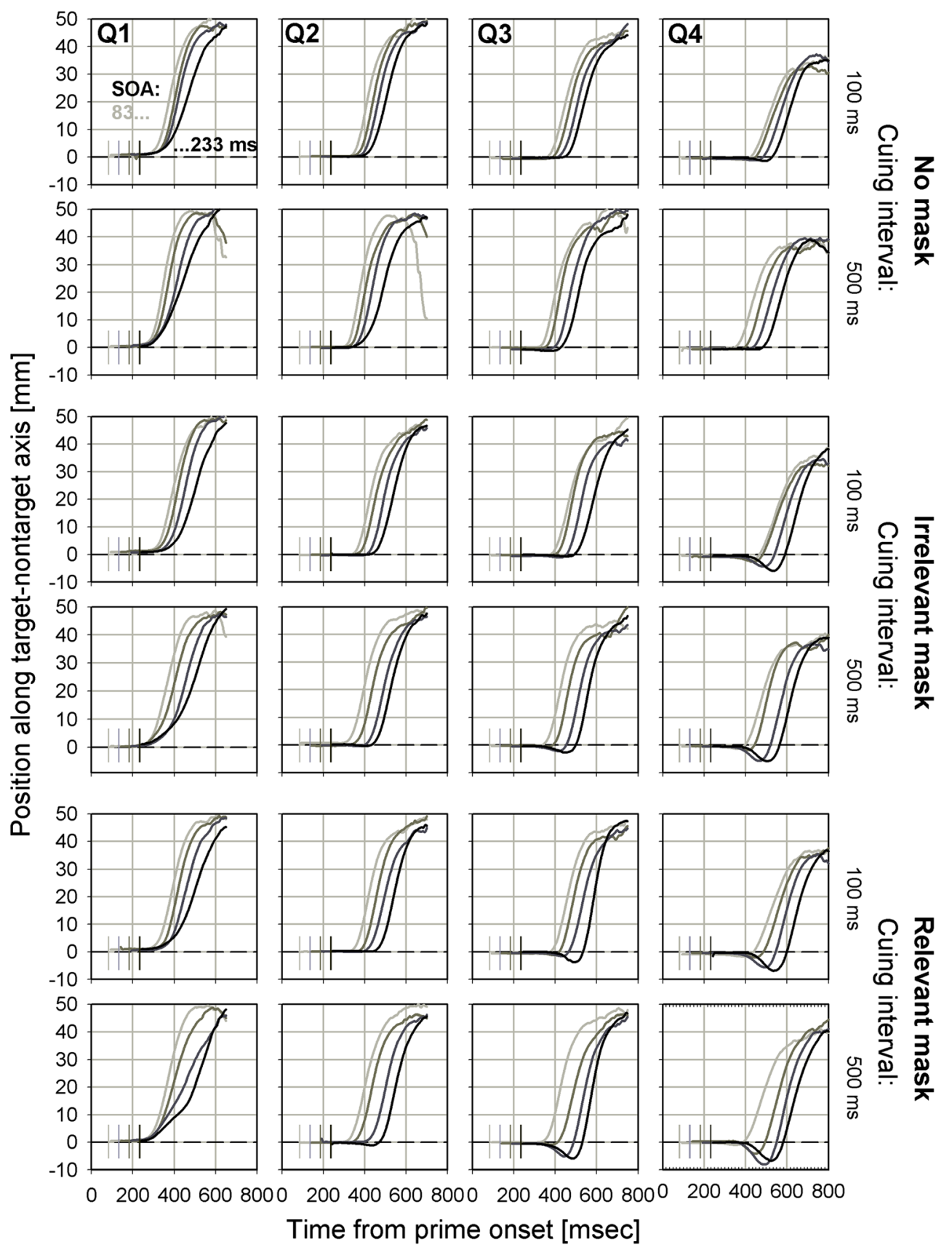

Fig. 6 Movement trajectories in Experiment 1 as a function of movement onset (quartiles: Q1-Q4). Only consistent trials are shown. Negative-going trajectories indicate movement in the direction opposite both the prime and target

discrimination performance, $\chi_{\mathrm{M}}^{2}(1)=255.85, p<.001$. Again, we observed an interaction of mask type and SOA, $\chi_{\mathrm{M} \times \mathrm{S}}^{2}(1)=8.60, p=.003$, this time reflecting a significant decrease of performance with SOA when the mask was relevant, but no such change for irrelevant or absent masks. Separate analyses of the three masking functions showed that they were all significantly different from chance, all $\chi_{\text {int }}^{2}(1) \geq$ 12.75 , all $p \mathrm{~s}<.001$, and that only for the relevant-mask condition did performance depend on prime-target SOA, $\chi_{\mathrm{S}}^{2}(1)$ $=8.31, p=.004$. This unexpected finding suggests that for response-relevant masks, the NCE can increase with prime- target SOA even though prime discrimination performance decreases (double dissociation; T. Schmidt \& Vorberg, 2006). Overall, the findings suggest that the NCE is unrelated to visual awareness of the prime (Jaśkowski, 2007b; Verleger et al., 2004).

\section{Discussion}

Our data reveal the major cause of the NCE: Response inhibition in the NCE is in fact a massive counterforce to the primed response, starting $350 \mathrm{~ms}$ after mask onset and 


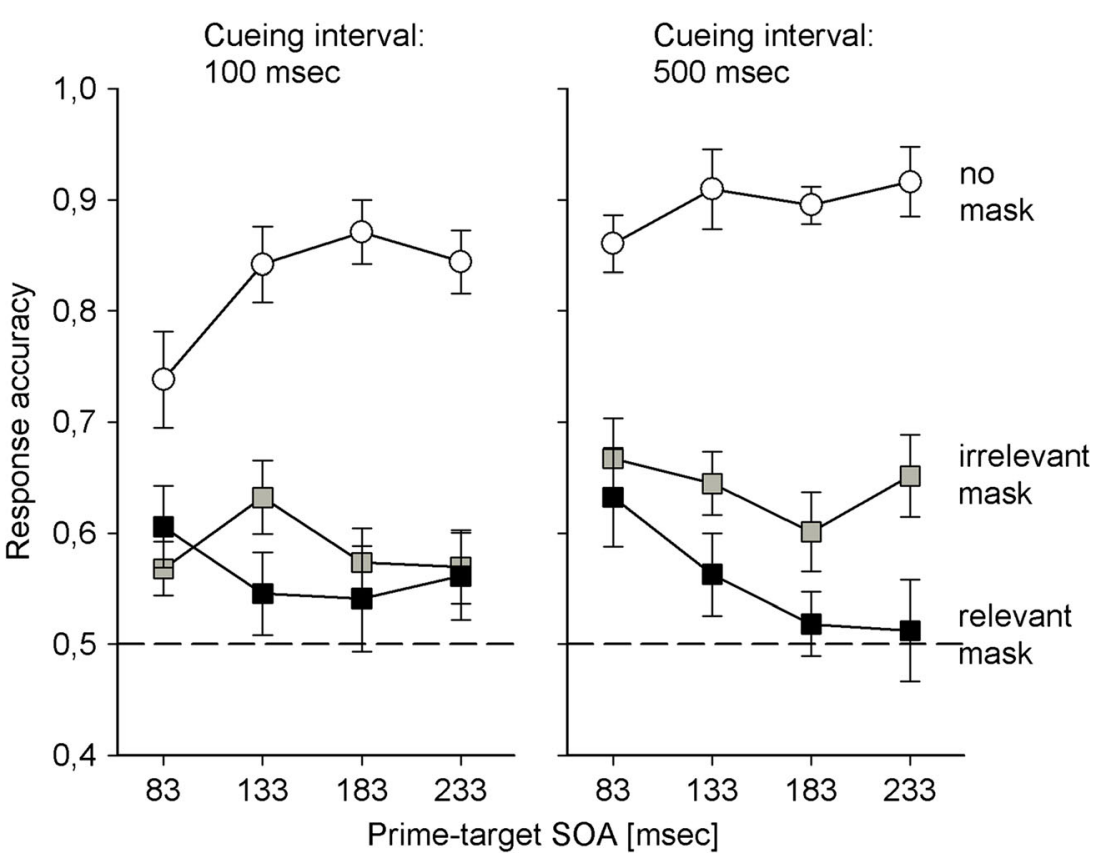

Fig. 7 Prime discrimination performance in Experiment 1

working precisely contrary to the prime direction. In consistent trials, this force is not only able to abolish the large positive priming effect, but actually drives the movement in a direction directly opposite to that indicated by every stimulus in the trial. Let us look at the effects of response activation and inhibition in more detail.

Only regular, positive priming effects occur in the absence of a mask between the prime and target onsets, even if the prime-target SOA is long. As has been observed in many other studies before, our data indicate that priming occurs because primes inconsistent with the target activate the incorrect response, whereas consistent primes activate the correct response. The resulting motor conflict in inconsistent trials leads to a delay of the pointing response, or even a slight detour of the movement in the wrong direction (T. Schmidt, 2002; T. Schmidt et al., 2006; cf. Song \& Nakayama, 2009). When the prime-target SOA becomes longer, this motor conflict deepens, because the misleading prime has progressively more time to activate the wrong response, and priming effects in response times as well as error rates become larger (Mattler \& Palmer, 2012; Schubert et al., 2013; Vorberg et al., 2003).

Tracing the time course of pointing responses allows us to trace the development of the NCE. It is surprising that the NCE is seen much more clearly in the early time course of the spatial priming effect than in the finger's arrival time (the equivalent of a finished keypress response). It is more pronounced for response-relevant than for irrelevant masks (Jaśkowski \& Ślósarek, 2007; Lleras \& Enns, 2004), and it is more pronounced at the longer cueing interval, validating Sumner et al.'s (2006) claim that the NCE is boosted by visual attention, just as the positive priming effects in the no-mask condition are (F. Schmidt \& Schmidt, 2010; T. Schmidt \& Seydell, 2008). Moreover, Fig. 5 reveals that the NCE is biphasic: An early, positive priming component dominates the response at shorter SOAs, and a later, negative component dominates the response at longer SOAs - with a gradual transition between those patterns as the SOA grows longer. Figure 6 reveals that this second, negative component reflects direction-specific inhibition of the primed response, which primarily affects slow responses (confirming the findings by Ocampo \& Finkbeiner, 2013). This specific inhibitory force occurs for any type of mask, and is thus not explained by feature-based priming from the mask elements. It is lifted by the onset of the target, but independent of cueing interval, and thus seems not to be boosted by visual attention. Importantly, it is almost (but not completely) abolished when no masks are presented between the primes and targets. These results are in remarkable correspondence with the mask-triggered inhibition account advocated by Jaśkowski and PrzekorackaKrawczyk (2005; Jaśkowski, 2007a). However, instead of their metaphor of an "emergency brake" triggered by the mask, we prefer the image of a rather powerful thrust reversal of the primed response.

Aside from this unexpected finding of a strong inhibitory counterforce to the primed response, can the response activations by prime, mask, and target stimuli be viewed as a strict sequence of stimulus-triggered motor events? Our data show that this is not the case. Even in the absence of any mask, when priming effects are strongly positive, their time course violates the chase criteria, and is thus not consistent with a simple feedforward system (T. Schmidt et al., 2006). The earliest phase of spatial priming functions is therefore not 
determined solely by the prime information. As a result, the priming functions fan up right from the time of movement onset (cf. Fig. 2c) instead of being initially invariant (cf. Fig. 2b). This implies that by the time of movement onset, information from the prime, mask, and target has already been integrated, and the response is no longer determined by a strict sequence of events.

Whenever masks are presented, they strongly reduce prime identification performance, thus leading to efficient backward masking. Note that NCEs are observed even under conditions in which prime discrimination performance is low $(50 \%-$ $60 \%$ ), and that the time course of the NCE with respect to prime-target SOA can even be opposite to that of prime discrimination, consistent with the view that visual awareness of the prime is not necessary for the NCE to occur (Jaśkowski, 2007b).

\section{Experiment 2}

Experiment 1 showed that the NCE cannot be explained by a strict sequence of feedforward activations induced in turn by primes, masks, and targets. Even when no mask is presented and priming effects are strongly positive, they fail to conform to the independence criterion of rapid-chase theory. Clearly, when the pointing response starts, some integration of the prime and target information has already occurred.

It remained to be shown that the failure to meet the chase criteria was not due to any details of the employed paradigm - for instance, the stimuli employed, the presence of attentional cues, or the presence of masks. Experiment 2 was designed to demonstrate that the time course of positive response priming would conform to the chase criteria when the same sequence of primes, masks, and targets was employed as in Experiment 1, adjusted to the shorter SOA range necessary for regular response priming.

\section{Method}

Participants Eight students from the University of Kaiserslautern (21-22 years of age, three of them male, all of them right-handed) volunteered for a payment of $€ 28$. Their vision was normal or corrected to normal, and all of them gave informed consent.

Apparatus and stimuli The apparatus and stimuli were the same as in Experiment 1. The procedure was also identical to the one in that experiment, with the following exceptions. We used only a single cueing interval of $500 \mathrm{~ms}$ and shorter intervals for the prime-target SOA $(33,50,67$, and $83 \mathrm{~ms}$, chosen to cover the full time range of response priming, while avoiding the long SOAs that would lead to an NCE). The masks were always presented $17 \mathrm{~ms}$ before target onset so that the prime could have an undisturbed impact on the motor response for a time similar to Experiment 1. All stimulus combinations of consistency, prime-target SOA, and mask type occurred equiprobably and pseudorandomly in a completely crossed repeated measures design (25 repetitions of each combination per participant and session, allowing us to obtain reliable data patterns on the level of individual participants). In each trial, correct target location was drawn randomly from a uniform distribution. For this experiment, the responsesensitive area around the target positions was slightly widened from 17 to $20 \mathrm{~mm}$ radius, to reduce the number of misrecorded responses. The arrival time was redefined as the point in time when the stylus first entered this sensitive area.

Participants performed three 1-h sessions of the Target ID task, followed by a $1-\mathrm{h}$ session of the Prime ID task, each consisting of one practice block followed by 20 blocks of 30 trials.

Statistical methods One Target ID session of one participant was lost due to equipment malfunction. Trials in the Target ID task were excluded if arrival times were shorter than $100 \mathrm{~ms}$ or longer than $1,000 \mathrm{~ms}$ ( $1.07 \%$ of trials). In addition, trials were excluded when participants failed to respond or when they hit one of the invalid target locations (1.07\% of trials in Target ID, $4.16 \%$ in Prime ID). Otherwise, the data were treated as in Experiment 1.

\section{Results}

The overall pattern of results differed strongly from that in Experiment 1. First, the priming effects in all masking conditions conformed to the chase criteria, such that the time course of the trajectorial priming function was initially invariant, depending only on the prime but not the mask characteristics. Second, large priming effects were obtained even in the presence of masks, and even though the masks led to strong reductions in prime identification performance. Our basic analysis consisted of an analysis of variance with the factors Consistency (C) and Prime-Target SOA (S).

Target ID: No masks In the absence of masks, arrivals occurred later in inconsistent than in consistent trials $\left[F_{\mathrm{C}}(1,7)=\right.$ $178.83, p<.001]$, and inconsistent trials produced more response errors $\left[F_{\mathrm{C}}(1,7)=10.78, p=.013\right]$ (Figs. 8, 9 and 10, left panels). These priming effects increased with increasing prime-target SOA in arrival times $\left[F_{\mathrm{C} \times \mathrm{S}}(3,21)=13.37, p<\right.$ $.001]$, as well as in error rates $\left[F_{\mathrm{C} \times \mathrm{S}}(3,21)=4.18, p=.018\right]$. Error rates generally increased with increasing prime-target $\operatorname{SOA}\left[F_{\mathrm{S}}(3,21)=3.32, p=.041\right]$.

The peak amplitude of the spatial priming effect $\left[F_{\text {int }}(1,7)\right.$ $=216.84, p<.001]$ was larger $\left[F_{\mathrm{S}}(1,7)=12.19, p<.001\right]$ and was reached later $\left[F_{\mathrm{S}}(1,7)=8.77, p=.003\right]$ with increasing prime-target SOAs (Fig. 10). All trajectorial priming 

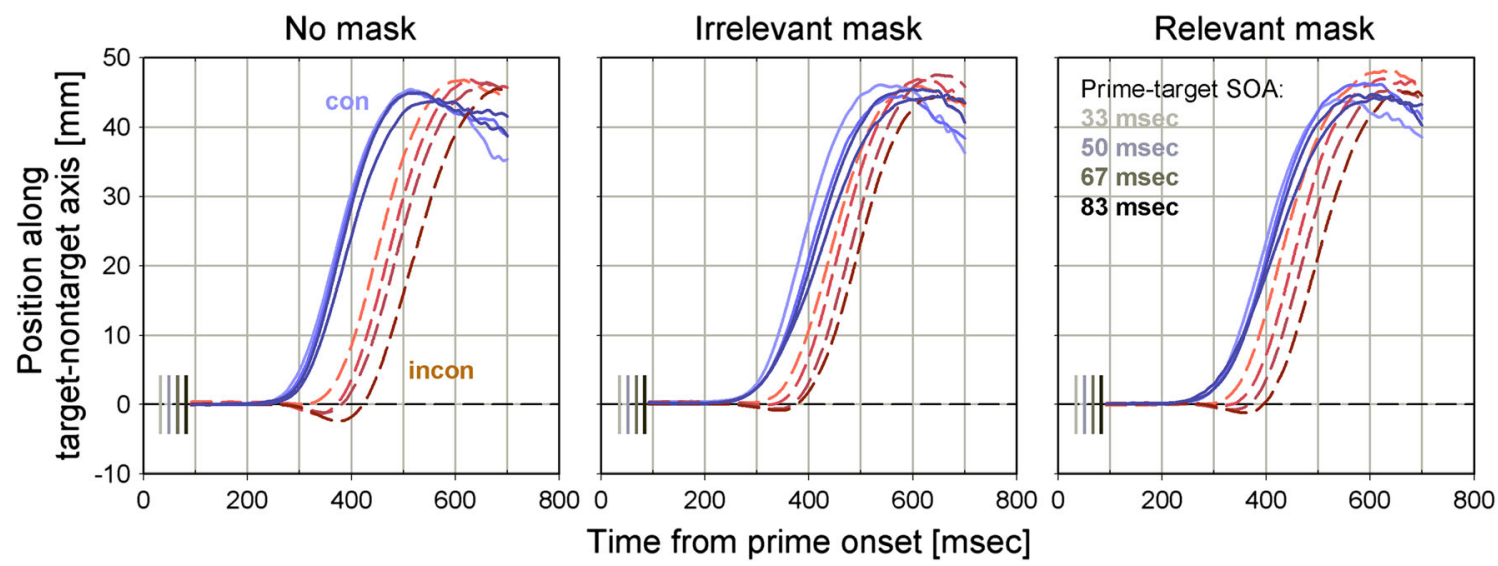

Fig. 8 Movement trajectories in Experiment 2, target identification task

functions shared the same initial time course, conforming to the predictions for a rapid-chase process (cf. Fig. 2b). The initial pointing response was thus controlled exclusively by the prime and not influenced by the target information.

Target ID: Irrelevant masks In the following, note that because the masks were presented $17 \mathrm{~ms}$ before the targets, any variation of prime-target SOA also changed the prime-mask SOA. Also note that two of the prime-mask SOAs in Experiment 2 were shorter than the SOA in Experiment 1 (either 17, 33, 50, or $67 \mathrm{~ms}$, as compared to Exp. 1's fixed $50 \mathrm{~ms}$ ), rendering the new experiment an even stricter test of the chase criteria.

Priming effects were smaller than in the absence of masks, but large priming effects were still obtained (Figs. 8, 9 and 10, middle panels). The disruptive effect of the masks was thus much smaller than in Experiment 1, even when the primemask SOA was shorter. With response-irrelevant masks, arrivals occurred later in inconsistent than in consistent trials $\left[F_{\mathrm{C}}(1,7)=102.89, p<.001\right]$, and inconsistent trials produced more response errors $\left[F_{\mathrm{C}}(1,7)=6.86, p=.034\right]$ (Fig. 9, middle panels). These priming effects were not significantly modulated by prime-target SOA. Error rates increased with increasing prime-target SOA $\left[F_{\mathrm{S}}(3,21)=7.56, p=.003\right]$. The peak amplitude of the spatial priming effect $\left[F_{\text {int }}(1,7)=\right.$ $189.56, p<.001]$ was not significantly modulated by primetarget SOA, but it was reached earlier at shorter prime-target SOAs $\left[F_{\mathrm{S}}(3,21)=4.56, p=.013\right]$ (Fig. 10, middle panel).

Target ID: Relevant masks With response-relevant masks, arrivals occurred later in inconsistent than in consistent trials $\left[F_{\mathrm{C}}(1,7)=123.41, p<.001\right]$, and inconsistent trials produced

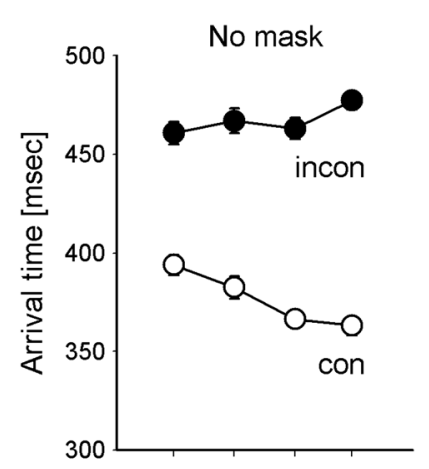

Cueing interval: $\mathbf{5 0 0} \mathbf{~ m s}$
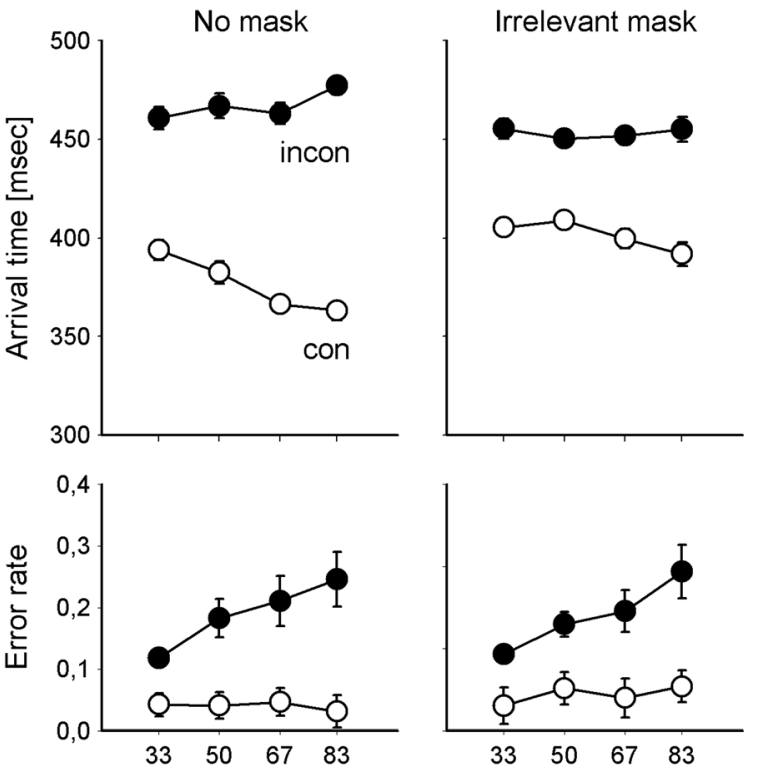

Relevant mask
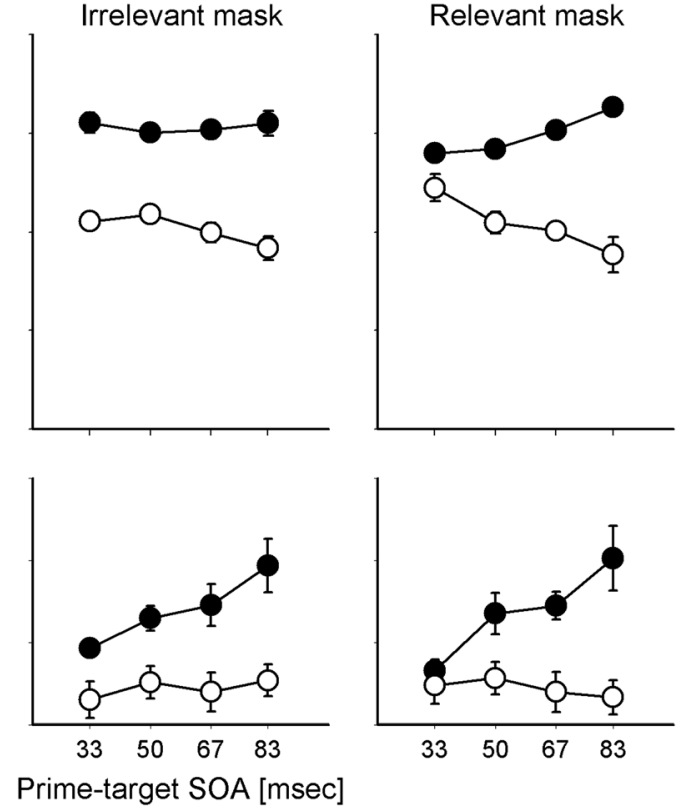

Fig. 9 Arrival times (upper panels) and error rates (lower panels) in Experiment 2, target identification task 


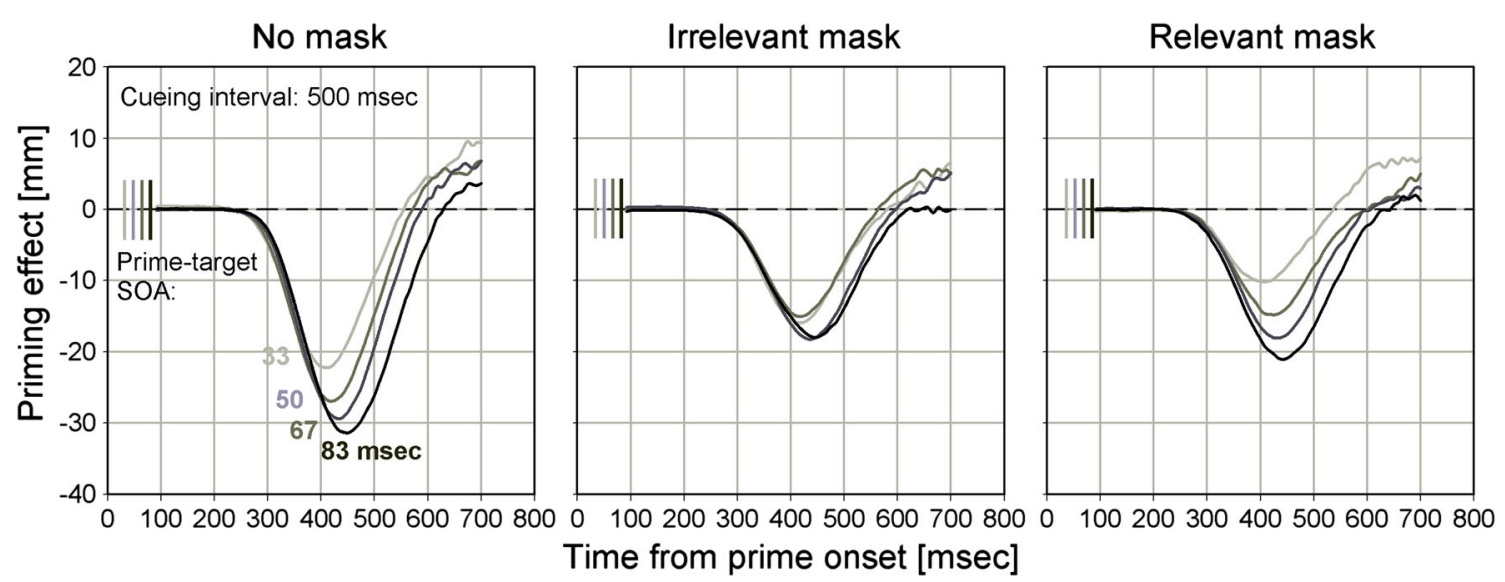

Fig. 10 Trajectorial priming functions in Experiment 2, target identification task

more response errors $\left[F_{\mathrm{C}}(1,7)=7.44, p=.029\right]$ (Fig. 9, right panels). These priming effects increased with prime-target SOA in arrival times $\left[F_{\mathrm{C} \times \mathrm{S}}(3,21)=12.15, p<.001\right]$ as well as in error rates $\left[F_{\mathrm{C} \times \mathrm{S}}(3,21)=6.81, p=.002\right]$. Error rates generally increased with increasing prime-target $\mathrm{SOA}\left[F_{\mathrm{S}}(3\right.$, $21)=4.57, p=.013]$. The peak amplitude of the spatial priming effect $\left[F_{\text {int }}(1,7)=120.24, p<.001\right]$ increased with primetarget SOA $\left[F_{\mathrm{S}}(3,21)=15.13, p<.001\right]$ and was reached earlier with shorter prime-target $\operatorname{SOAs}\left[F_{\mathrm{S}}(3,21)=3.62, p\right.$ $=.030]$ (Fig. 10).

To compare the different masking conditions, we performed an additional analysis of variance with the factors Mask Type (M) and Prime-Target SOA, with priming effects on arrival times as the dependent variable. Priming effects were significantly different for the different mask types $\left[F_{\mathrm{M}}(2,14)=33.99, p<.001\right]$ and increased with prime-target SOA $\left[F_{\mathrm{S}}(3,21)=29.63, p<.001\right]$, and a slight interaction between the factors reflected the reduced slope of the priming effect in the irrelevant-mask condition $\left[F_{\mathrm{M} \times \mathrm{S}}(6,42)=2.25, p\right.$ $<.057]$. In the error rates, we found significant effects of mask type and SOA, but no interaction. When the analysis was restricted to the conditions with masks present, no main effect of mask emerged, but a main effect of SOA was still present $\left[F_{\mathrm{S}}(3,21)=12.50, p=.002\right]$, and the interaction was more pronounced $\left[F_{\mathrm{M} \times \mathrm{S}}(3,21)=3.59, p=.031\right]$. In the error rates, only the main effect of SOA was significant.

Direction-specific counterforce production To assess a possible effect of counterforce production in consistent trials, we performed the same quartile split and integral test (area under the negative-going part of the trajectorial priming function) as in Experiment 1. The results are shown in Fig. 11, which indicates that responses in the antiprime direction generally do not occur in Quartiles 1-3, but some subtle negativegoing deflections appear in the slowest responses (Quartile 4). An analysis of variance with the factors Onset Quartile (Q) and Prime-Target SOA revealed some evidence for significant area effects in the no-mask and irrelevant-mask conditions, but not in the relevant-mask condition $\left[F_{\text {int }} \mathrm{s}(1\right.$, $7)=4.78,13.62$, and $3.02, p s=.065, .008$, and .126 , respectively]. In the no-mask condition, the area tended to increase with quartile $\left[F_{\mathrm{Q}}(3,21)=3.02, p=.053\right]$. All of these effects were small, relative to those in Experiment 1.

Prime ID Generally, prime discrimination performance was best with masks absent, and worse for the relevant and irrelevant masks (Fig. 12). Logistic regression with the factors Prime-Target SOA and Mask Type (standardized) confirmed a significant effect of mask type, Wald's $\chi_{\mathrm{M}}^{2}(1)=182.87, p<$ .001 . Separate analyses of the three masking functions showed that performance was different from chance in the no-mask condition, $\chi_{\text {int }}^{2}(1)=376.92, p<.001$, as well as in the irrelevant-mask condition, $\chi_{\text {int }}^{2}(1)=15.87, p<.001$, but not in the relevant-mask condition, $\chi_{\text {int }}^{2}(1)=0.13, p=.929$. The SOA effects were nonsignificant in all three cases.

\section{Discussion}

Experiment 2 demonstrates that the task design and stimulus set employed in Experiment 1 was able to produce regular, positive response priming effects even under conditions of strong backward masking: Priming was observed in many conditions in which prime discrimination performance was close to chance. The most important issue, however, was whether the time course of priming would conform to the chase criteria (T. Schmidt et al., 2006).

In the absence of masks, all of the chase criteria were met: The onset time and initial direction of the pointing response were determined by the prime, not the target; the target was typically able to reverse the prime-driven response in midflight; and most importantly, the initial time course of the pointing response was invariant when time-locked to prime onset. This pattern suggests that as long as only the prime and target have to be considered, the initial time course of the response is controlled exclusively by the prime and independent of the onset time of the target. 


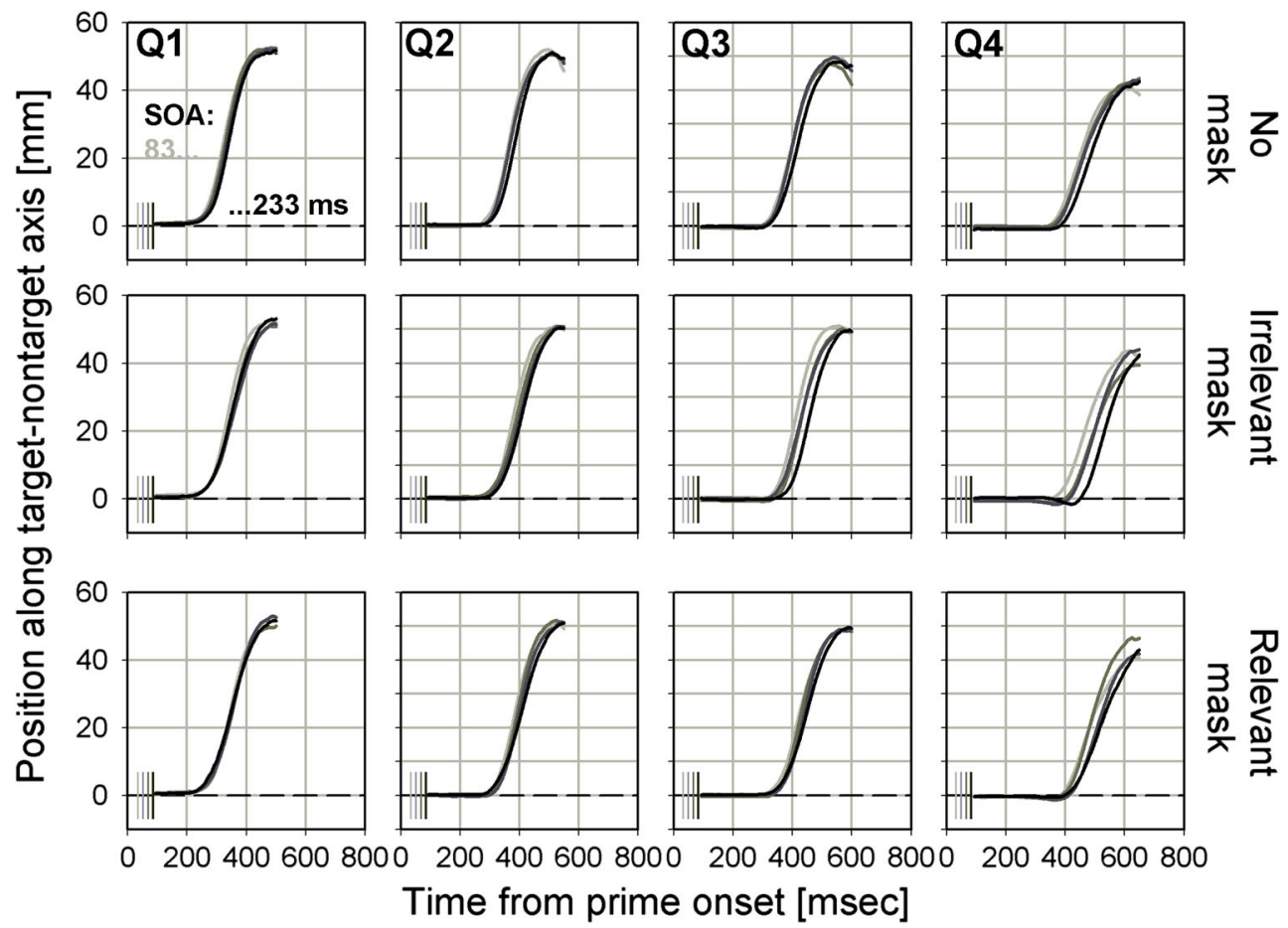

Fig. 11 Movement trajectories in Experiment 2 as a function of movement onset (quartiles: Q1-Q4). Only consistent trials are shown. Negative-going trajectories indicate movement in the direction opposite both the prime and target

The chase criteria were also met when response-irrelevant masks or response-relevant masks were presented. However, the characteristics of the priming effects differed markedly between relevant and irrelevant masks. One major difference seems to have been that relevant masks reduced the priming effects selectively at short SOAs, which was evident not only in arrival times but also in spatial priming effects. This would be expected if the antiprime elements in the mask triggered the

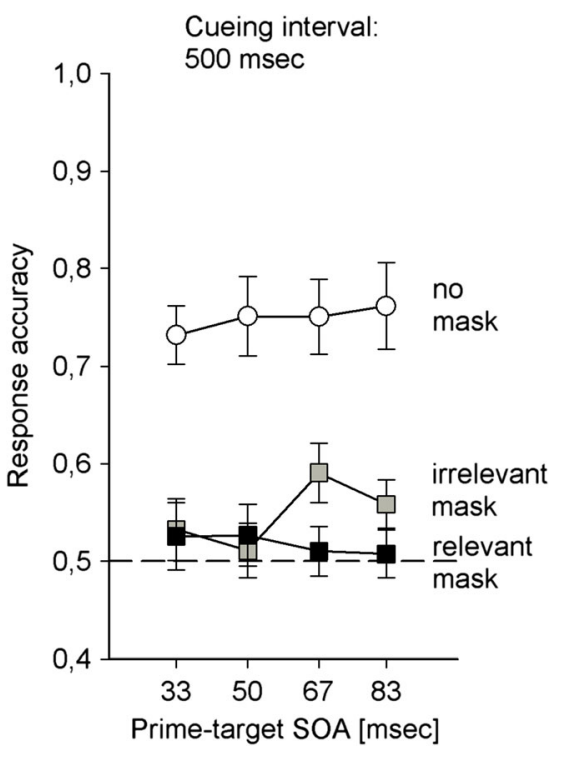

Fig. 12 Prime discrimination performance in Experiment 2 response opposite to that triggered by the prime - thereby providing a quick thrust of incorrect response activation in consistent trials, and an earlier start of correct response activation (effectively, a shorter prime-target SOA) in inconsistent trials. When the prime-target SOA was long, the impact of the mask on response times was expected to be weaker because the prime has had more time to activate the response.

Under all masking conditions, the spatial priming functions were tightly locked to prime onset, and because the SOA between the mask and target was constant, the rapid-chase pattern implies that the earliest priming effects were independent of the onset time not only of the targets, but of the masks as well. However, the presence of masks had a clearly detrimental effect on the time course of the spatial priming effect. Whenever masks were present, responses in consistent trials accelerated less rapidly, reached lower peak velocity, and reached the correct target later than when masks were absent. In inconsistent trials, detours in the incorrect direction were less pronounced, ended sooner, and reached the correct target sooner. Clearly, the masks were able to affect the ongoing pointing response, supporting the notion that the masks have an active role in the motor process after initiating a processing sweep of their own (Jaśkowski, 2007a, b; Jaśkowski \& Ślósarek, 2007; Lleras \& Enns, 2004). The ability of the masks to reduce the ability to discriminate the prime cannot explain its impact on the motor response, because that reduction was too strong: Large priming effects were still observed when prime discrimination was close to chance level. 
There is a puzzling aspect of these data: The masks clearly affect the early time course of the pointing movements, but they do so without disturbing the rapid-chase properties. In a feedforward system, this can only be explained by interference between the masks and the prime before the masks themselves exert an influence on the response. This mechanism might be explained in the same way that visual backward masking is explained by a disruption of reentrant activity (DiLollo, Enns, \& Rensink, 2000; Lamme, Zipser, \& Spekreijse, 2002; cf. Bachmann, 1984, 2007). Specifically, when a prime is presented without a mask, its feedforward sweep is followed by a trail of consonant activity that is already based on recurrent processing. When a mask is presented shortly after the prime, this trail of supplanting information is cut short because the mask breaks the recurrent loop, curtailing the effective duration of the prime signal and thus reducing its signal strength. If this occurs before the mask's feedforward sweep starts to influence the motor response, consistent and inconsistent primes would be affected identically, leaving the rapid-chase properties intact.

\section{General discussion}

Our aim in this study was to get insight into the time courses of response activation and inhibition in two complementary paradigms: response priming and the NCE. In particular, we wanted to know whether the NCE can be explained as a strict sequence of response activation and inhibition events timelocked to the visual stimuli, and whether the NCE could be explained with the same simple feedforward model suitable for explaining response priming. Indeed, our data provide insight into the time course of the NCE as compared to regular, positive response priming. As has been shown in many previous studies, regular response priming is consistent with a simple feedforward model: Primes and targets elicit sequential sweeps of feedforward activity that take control over the pointing response in strict sequence. Therefore, primed pointing movements conform to the chase criteria: Primes initiate the response and have exclusive control over it for a time depending on prime-target SOA before masks and targets start influencing the response. This is not the case in the NCE: Right from the start of the overt movement, the response is controlled jointly by prime, mask, and target information. Purely sequential models are thus able to account for the positive response priming effect, but not for the NCE. In the following, we describe the processes involved in the NCE. It turns out that two important new findings have to be considered: (1) The presence of a massive inhibitory force in precise counterdirection to the primed response; and (2) the integration of prime, mask, and target information over time, which introduces a component of information persistence that has not been considered by any previous theory of the effect.
Let us start from a general description of the regular response priming process. The most successful models of response priming (Mattler \& Palmer, 2012; Schubert et al., 2013; Vorberg et al., 2003) assume an accumulation of stimulus-related evidence toward one of two response thresholds: one associated with the correct response, the other with the incorrect response. Consistent and inconsistent primes first initiate accumulation toward the correct or incorrect response threshold that continues for as long as the prime-target SOA allows. Then, the target takes over the accumulation process and drives it toward the correct response threshold. Errors occur if an inconsistent prime is able to drive the process over the incorrect threshold, which is more likely to happen at longer prime-target SOAs. Consequently, we argue that in a typical NCE experiment with long prime-target SOAs, the response thresholds are likely to be high, leading to a timeconsuming accumulation process. These high thresholds are necessary to avoid responding to the prime instead of the target, which would incur an error rate of close to $50 \%$. High response thresholds have three important consequences: They keep down error rates, they delay responses, and they open the system up for effects of information persistence.

The existence of information persistence in the NCE can be inferred from two pieces of evidence: First, responses are completed relatively long after prime onset (about $700 \mathrm{~ms}$, despite error rates of up to $20 \%$ ), and second, the initial response is controlled jointly by the prime, mask, and target information, in contrast to the strictly sequential response control observed in the regular response priming effect. (Indeed, our analysis of response onset times in Exp. 1 showed that participants always wait out the target and do not start their responses before target onset.) Information persistence greatly complicates any theoretical account of the NCE, because it introduces a memory component: Instead of being triggered by an incoming feedforward sweep, the response has to be started out of a memory buffer. ${ }^{5}$ This buffer must contain at least the following types of information: (1) prime information, because the NCE depends on the identity of the prime, and (2) mask information, because masks interfere with the response process. It possibly also contains target information, although the fact that arrival times relative to the target were similar between Experiments 1 and 2 suggests that the response is only delayed until the target's feedforward sweep

\footnotetext{
${ }^{5}$ Such a buffer is not necessarily a distinct boxological unit, but merely a modeling device that accounts for the time difference between immediate and delayed responses and describes the fates of the prime, mask, and target information until the time of response onset. Its properties may all be explained by information persistence in familiar parts of the visuomotor system - for example, an integration phase in the buildup of motor activity. Note that our notion of information persistence is similar to Klauer and Dittrich's (2010) idea that the information from prime, mask, and target features is sampled during an "evaluation window" starting at mask onset. This entails the idea of information integration over time and might be equivalent to assuming a short-term buffer.
} 
arrives. Previous research has shown that performing motor responses out of memory can have a strong impact on the type of processing involved (Goodale, 2011).

How would a memory buffer contribute to the NCE? At the time of response onset, the buffer may hold prime features (from primes and relevant masks), antiprime features (from relevant masks), and neutral features not mapped to any response (from irrelevant masks). Prime and antiprime features bias the decision process toward the correct or incorrect response, whereas neutral features dilute the impact of response-relevant features. When the mask is absent, only prime information enters the buffer, and its capacity for priming the response to the target depends on the yet unknown interplay of information accrual and decay in the buffer. In any event, the buffer will promote positive priming. In contrast, when the prime is followed by a response-relevant mask, the buffer is filled with antiprime features on top of prime features, and the priming ability of the buffer depends on the relative strength of those features at the end of the mask-target SOA. If antiprime features are more prominent than prime features, negative priming ensues. Finally, when the prime is followed by a neutral mask, the prime information is drowned out by neutral features that are not mapped to any motor response, but the residue may still lead to small positive priming effects. All of this might be regarded as a memory variant of the objectupdating or active-mask accounts (Lleras \& Enns, 2004; Verleger et al., 2004).

But our findings clearly show that mask features only have a modulatory influence on the NCE: The major driving force of the effect is a massive, spatially directed response inhibition of the primed response. This inhibition is time-locked to the mask, is lifted by the onset of the target, does not seem to depend on mask type or the state of visual attention, and is almost absent when no masks are presented, suggesting that it is basically a case of mask-triggered inhibition (Jaśkowski, 2007a; Jaśkowski \& Przekoracka-Krawczyk, 2005). Importantly, this inhibitory component is large enough to explain how any mask is able to abolish the large positive response priming effect occurring when no mask is present. The effects of mask features and of visual attention only seem to modulate what is left of the priming effect when response inhibition has taken place. Mask features are able to give the residual effect a small positive or negative bias, and visual attention can boost these stimulus-driven priming effects, but the major work in the NCE is done by thrust reversal of the prime-initiated response.

Our findings shed new light on the previous evidence for response inhibition in the NCE. Klauer and Dittrich (2010) showed that an NCE can be obtained even in the absence of any mask between prime and target, leaving only response inhibition as a viable explanation. Similarly, Vorberg (2005) has demonstrated an NCE with stimuli not containing any antiprime features. ${ }^{6}$ He presented a small arrow as a prime, followed by a metacontrast mask after an SOA of $70 \mathrm{~ms}$. After the mask, a large arrow target at the same position was presented only in outline, so that it did not have any masking effect itself. The trick of the experiment was in the variation of the mask. The outer contour of the mask was always neutral, but the inner cutout of the mask (leading to the metacontrast masking of the prime) was either neutral or identical to the prime. For instance, if the prime pointed to the left, it was followed by a mask of a neutral outline shape with a central cutout also pointing to the left. Of course, such a mask gives away the identity of the prime, but it contains no antiprime features whatsoever. Control conditions without primes separated the prime-induced effects from any effects of the response-relevant mask. Crucially, Vorberg (2005) found an NCE even in the absence of antiprime features, ruling out any explanation solely in terms of an active-mask or object substitution account. Note the similarity of those findings to the paradoxical movements presented in Fig. 6.

Several of our findings are of methodological importance. First, response inhibition does not seem able to influence the response if the response occurs too quickly. Our findings support Ocampo and Finkbeiner's (2013) conclusion that the NCE is limited to the slower responses, and our estimate is that response inhibition is in full force only as late as $400 \mathrm{~ms}$ after mask onset. Any further insight into these inhibitory mechanisms could only be gained by splitting responses by their speed of onset, as was suggested by those authors. Second, if priming effects are only evaluated at the time when the response is complete (i.e., at the arrival or keypress time), the most interesting phase of the effect is missed: the time when a large response-activating force induced by the prime clashes with a mask-triggered counterforce of about the same magnitude. Explaining the NCE not only requires explaining the net priming effect remaining at the end, but the much more dramatic kinetic processes happening before. Third, our paradigm liberates the NCE from the cramped confines of twointerval yes-no keypress tasks. Our data show that an NCE can be obtained without a predetermined mapping between the identity of a single target and an associated response. In our paradigm, once the spatial cue has been processed, the task boils down to a two-alternative forced choice, but one in which the correct target can be at one of ten possible locations. Because the set of conflicting responses has to be set up on the fly upon processing of the cue, the NCE cannot depend on a preestablished pair of stimulus-response channels.

What is the role of attention in the NCE? We found that attention modulates the positive response-priming effect ( $\mathrm{F}$.

\footnotetext{
${ }^{6}$ In an unpublished study that has been presented at several conferences. A slideshow of this experiment (in the German language, but selfexplanatory) may be available from Dirk Vorberg, University of Münster, Germany.
} 
Schmidt \& Schmidt, 2010; T. Schmidt \& Seydell, 2008) as well as the NCE (Sumner et al., 2006). In our paradigm, spatial selection of the cued locations is necessary to obtain a spatially directed priming effect in the first place, and our observation of such effects implies that spatial selection must have taken place. However, this deployment of attention does not seem to modulate the specific response inhibition component that is mainly responsible for the NCE. It might be that the magnitude of this inhibition is independent of attention.

Finally, what is the role of visual awareness in the NCE? Both positive and reverse priming effects occur under conditions of strong visual masking. For regular, positive response priming, it has often been shown that motor activation by the prime can remain intact when the prime cannot be discriminated above chance accuracy (e.g., F. Schmidt \& Schmidt, 2010; Vorberg et al., 2003). Moreover, response priming can vary independently of discrimination performance: Priming can increase with prime-target SOA, while the ability to discriminate the prime decreases (Albrecht, Klapötke, \& Mattler, 2010; Mattler, 2003; Vorberg et al., 2003). The present data suggest the same property for the NCE: It occurs under substantial masking (Verleger et al., 2004), as well as with clearly visible primes (Jaśkowski, 2007b; Klauer \& Dittrich, 2010), and it seems to build up with increasing SOA, while prime discrimination performance is decreasing. This double dissociation between the priming effect and discrimination of the prime strongly suggests that awareness of the prime cannot explain the time course of the NCE (T. Schmidt, 2007; T. Schmidt \& Vorberg, 2006). It also suggests that response inhibition of the prime is not due to the mask's ability to suppress visual awareness of the prime (Eimer \& Schlaghecken, 1998, 2002, 2003; Schlaghecken \& Eimer, 2002), but rather to the motor processes induced by the masks (Jaśkowski, 2007a; Jaśkowski \& Przekoracka-Krawczyk, 2005).

Author note This work was conducted during our time in Karl Gegenfurtner's lab at the University of Giessen, Germany. We thank Karl for his support, and Anna Seydell-Greenwald, Anke Haberkamp, Andreas Weber, and Bruno Breitmeyer for helpful discussions. Many thanks to Sven Panis for discussions and corrections of the manuscript, and to Katharina Oßwald for collecting some of the data. Part of this work was supported by the German Research Foundation (DFG).

\section{References}

Albrecht, T., Klapötke, S., \& Mattler, U. (2010). Individual differences in metacontrast masking are enhanced by perceptual learning. Consciousness and Cognition, 19, 656-666. doi:10.1016/j.concog. 2009.12.002

Allport, A. (1989). Visual attention. In M. I. Posner (Ed.), Foundations of cognitive science (pp. 631-682). Cambridge, MA: MIT Press.

Allport, A., Styles, E. A., \& Hsieh, S. (1994). Shifting intentional set: Exploring the dynamic control of tasks. In C. Umiltà \& M. Moscovitch (Eds.), Attention and performance XV: Conscious and nonconscious information processing (pp. 421-452). Cambridge, MA: MIT Press.

Bachmann, T. (1984). The process of perceptual retouch: Nonspecific afferent activation dynamics in explaining visual masking. Perception \& Psychophysics, 35, 69-84.

Bachmann, T. (2007). Binding binding: Departure points for a different version of the perceptual retouch theory. Advances in Cognitive Psychology, 3, 41-55. doi:10.2478/v10053-008-0013-4

Boy, F., Husain, M., \& Sumner, P. (2010). Unconscious inhibition separates two forms of cognitive control. Proceedings of the National Academy of Sciences, 107, 11134-11139.

Braver, T. S. (2012). The variable nature of cognitive control: A dual mechanisms framework. Trends in Cognitive Sciences, 16, 106113. doi:10.1016/j.tics.2011.12.010

Breitmeyer, B. G., \& Öğmen, H. (2006). Visual masking. Oxford, UK: Oxford University Press.

Brenner, E., \& Smeets, J. B. J. (2004). Color vision can contribute to fast corrections of arm movements. Experimental Brain Research, 158, 302-307.

Bullier, J. (2001). Integrated model of visual processing. Brain Research Reviews, 36, 96-107.

Bullier, J. (2004). Communications between cortical areas of the visual system. In L. M. Chalupa \& J. W. Werner (Eds.), The visual neurosciences (Vol. 1, pp. 522-540). Cambridge, MA: MIT Press.

Chen, C.-M., Lakatos, P., Shah, A. S., Mehta, A. D., Givre, S. J., Javitt, D. C., \& Schroeder, C. E. (2007). Functional anatomy and interaction of fast and slow visual pathways in macaque monkeys. Cerebral Cortex, 17, 1561-1569. doi:10.1093/cercor/bh1067

Cousineau, D. (2005). Confidence intervals in within-subjects designs: A simpler solution to Loftus and Masson's method. Tutorials in Quantitative Methods for Psychology, 1, 42-46.

de Jong, R., Coles, M. G., Logan, G. D., \& Gratton, G. (1990). In search of the point of no return: The control of response processes. Journal of Experimental Psychology: Human Perception and Performance, 16, 164-182. doi:10.1037/0096-1523.16.1.164

Di Lollo, V., Enns, J. T., \& Rensink, R. A. (2000). Competition for consciousness among visual events: The psychophysics of reentrant visual processes. Journal of Experimental Psychology: General, 129, 481-507. doi:10.1037/0096-3445.129.4.481

Eimer, M., \& Schlaghecken, F. (1998). Effects of masked stimuli on motor activation: Behavioral and electrophysiological evidence. Journal of Experimental Psychology: Human Perception and Performance, 24, 1737-1747. doi:10.1037/0096-1523.24.6.1737

Eimer, M., \& Schlaghecken, F. (2002). Links between conscious awareness and response inhibition: Evidence from masked priming. Psychonomic Bulletin \& Review, 9, 514-520. doi:10.3758/ BF03196307

Eimer, M., \& Schlaghecken, F. (2003). Response facilitation and inhibition in subliminal priming. Biological Psychology, 64, 7-26. doi:10. 1016/S0301-0511(03)00100-5

Eriksen, B. A., \& Eriksen, C. W. (1974). Effects of noise letters upon the identification of a target letter in a nonsearch task. Perception \& Psychophysics, 16, 143-149. doi:10.3758/BF03203267

Goodale, M. A. (2011). Transforming vision into action. Vision Research, 51, 1567-1587. doi:10.1016/j.visres.2010.07.027

Grainger, J. E., Scharnowski, F., Schmidt, T., \& Herzog, M. H. (2013). Two primes priming: Does feature integration occur before response activation? Journal of Vision, 13(8), 19. doi:10.1167/13.8.19. 1-10.

Green, D. M., \& Swets, J. A. (1966). Signal detection theory and psychophysics. New York, NY: Wiley.

Jaśkowski, P. (2007a). The effect of nonmasking distractors on the priming of motor responses. Journal of Experimental Psychology: Human Perception and Performance, 33, 456-468. doi:10.1037/ 0096-1523.33.2.456 
Jaśkowski, P. (2007b). The negative compatibility effect with nonmasking flankers: A case for mask-triggered inhibition hypothesis. Consciousness and Cognition, 17, 765-777.

Jaśkowski, P., \& Przekoracka-Krawczyk, A. (2005). On the role of mask structure in subliminal priming. Acta Neurobiologiae Experimentalis, 65, 409-417.

Jaśkowski, P., \& Ślósarek, M. (2007). How important is a prime's gestalt for subliminal priming? Consciousness and Cognition, 16, 485497. doi:10.1016/j.concog.2006.06.005

Kirchner, H., \& Thorpe, S. J. (2006). Ultra-rapid object detection with saccadic eye movements: Visual processing speed revisited. Vision Research, 46, 1762-1776.

Klapp, S. T., \& Hinkley, L. B. (2002). The negative compatibility effect: Unconscious inhibition influences reaction time and response selection. Journal of Experimental Psychology: General, 131, 255-269. doi:10.1037/0096-3445.131.2.255

Klauer, K. C., \& Dittrich, K. (2010). From sunshine to double arrow: An evaluation window account of negative compatibility effects. Journal of Experimental Psychology: General, 139, 490-510.

Klotz, W., Heumann, M., Ansorge, U., \& Neumann, O. (2007). Electrophysiological activation by masked primes: Independence of prime-related and target-related activities. Advances in Cognitive Psychology, 3, 449-465.

Klotz, W., \& Neumann, O. (1999). Motor activation without conscious discrimination in metacontrast masking. Journal of Experimental Psychology: Human Perception and Performance, 25, 976-992. doi:10.1037/0096-1523.25.4.976

Klotz, W., \& Wolff, P. (1995). The effect of a masked stimulus on the response to the masking stimulus. Psychological Research, 58, 92 101.

Kunde, W., Kiesel, A., \& Hoffmann, J. (2003). Conscious control over the content of unconscious cognition. Cognition, 88, 223-242.

Lamme, V. A. F., \& Roelfsema, P. R. (2000). The distinct modes of vision offered by feedforward and recurrent processing. Trends in Neurosciences, 23, 571-579. doi:10.1016/S0166-2236(00)01657-X

Lamme, V. A. F., Zipser, K., \& Spekreijse, H. (2002). Masking interrupts figure-ground signals in V1. Journal of Cognitive Neuroscience, 14, 1044-1053.

Leuthold, H., \& Kopp, B. (1998). Mechanisms of priming by masked stimuli: Inferences from event-related brain potentials. Psychological Science, 9, 263-269.

Lingnau, A., \& Vorberg, D. (2005). The time course of response inhibition in masked priming. Perception \& Psychophysics, 67, 545-557.

Lleras, A., \& Enns, J. T. (2004). Negative compatibility or object updating? A cautionary tale of mask-dependent priming. Journal of Experimental Psychology: General, 133, 475-493. doi:10.1037/ 0096-3445.133.4.475

Logan, G. D., \& Cowan, W. B. (1984). On the ability to inhibit thought and action: A theory of an act of control. Psychological Review, 9, 295-327. doi:10.1037/0033-295X.91.3.295

Macmillan, N. A., \& Creelman, C. D. (2005). Detection theory: A user's guide (2nd ed.). Mahwah, NJ: Erlbaum.

Mattler, U. (2003). Priming of mental operations by masked stimuli. Perception \& Psychophysics, 65, 167-187. doi:10.3758/ BF03194793

Mattler, U., \& Palmer, S. (2012). Time course of free-choice priming effects explained by a simple accumulator model. Cognition, 123, 347-360. doi:10.1016/j.cognition.2012.03.002

Merigan, W. H., \& Maunsell, J. H. R. (1993). How parallel are the primate visual pathways? Annual Review of Neuroscience, 16, 369402

Milner, A. D., \& Goodale, M. A. (1995). The visual brain in action. Oxford, UK: Oxford University Press.

Neumann, O. (1990). Direct parameter specification and the concept of perception. Psychological Research, 52, 207-215.
Ocampo, B., \& Finkbeiner, M. (2013). The negative compatibility effect with relevant masks: A case for automatic motor inhibition. Frontiers in Psychology, 4, 822. doi:10.3389/fpsyg.2013.00822

Panis, S., \& Hermens, F. (2014). Time course of spatial contextual interference: Event history analyses of simultaneous masking by nonoverlapping patterns. Journal of Experimental Psychology: Human Perception and Performance, 40, 129-144.

Posner, M. I. (1980). Orienting of attention. Quarterly Journal of Experimental Psychology, 32, 3-25. doi:10.1080/ 00335558008248231

Praamstra, P., \& Seiss, E. (2005). The neurophysiology of response competition: Motor cortex activation and inhibition following subliminal response priming. Journal of Cognitive Neuroscience, 17, 483-493.

Rogers, R. D., \& Monsell, S. (1995). Costs of a predictable switch between simple cognitive tasks. Journal of Experimental Psychology: General, 124, 207-231. doi:10.1037/0096-3445.124.2.207

Roland, P. E. (2010). Six principles of visual cortical dynamics. Frontiers in Systems Neuroscience, 4, Article 28.

Schlaghecken, F., \& Eimer, M. (2002). Motor activation with and without inhibition: Evidence for a threshold mechanism in motor control. Perception \& Psychophysics, 64, 148-162. doi:10.3758/ BF03194564

Schlaghecken, F., \& Eimer, M. (2006). Active masks and active inhibition: A comment on Lleras and Enns (2004) and Verleger, Jaśkowski, Aydemir, van der Lubbe, and Groen (2004). Journal of Experimental Psychology: General, 135, 484-494. doi:10.1037/ 0096-3445.135.3.484

Schmidt, T. (2002). The finger in flight: Real-time motor control by visually masked color stimuli. Psychological Science, 13, 112118. doi:10.1111/1467-9280.00421

Schmidt, T. (2007). Measuring unconscious cognition: Beyond the zeroawareness criterion. Advances in Cognitive Psychology, 3, 275-287.

Schmidt, T. (2014). Behavioral criteria of feedforward processing in Rapid-Chase Theory: Some formal considerations. Retrieved from arXiv: $1405.5795 \mathrm{v} 2$ [q-bio.NC]

Schmidt, T., Haberkamp, A., Veltkamp, G. M., Weber, A., SeydellGreenwald, A., \& Schmidt, F. (2011a). Visual processing in rapidchase systems: Image processing, attention, and awareness. Frontiers in Psychology, 2, 169. doi:10.3389/fpsyg.2011.00169

Schmidt, F., Haberkamp, A., \& Schmidt, T. (2011b). Dos and don'ts in response priming research. Advances in Cognitive Psychology, 7 , $120-131$.

Schmidt, T., Niehaus, S., \& Nagel, A. (2006). Primes and targets in rapid chases: Tracing sequential waves of motor activation. Behavioural Neuroscience, 120, 1005-1016.

Schmidt, T., \& Schmidt, F. (2009). Processing of natural images is feedforward: A simple behavioral test. Attention, Perception, \& Psychophysics, 71, 594-606. doi:10.3758/APP.71.3.594

Schmidt, F., \& Schmidt, T. (2010). Feature-based attention to unconscious shapes and colors. Attention, Perception, \& Psychophysics, 72, 1480-1494. doi:10.3758/APP.72.6.1480

Schmidt, F., \& Schmidt, T. (2014). Rapid processing of closure and viewpoint-invariant symmetry: Behavioral criteria for feedforward processing. Psychological Research, 78, 37-54.

Schmidt, T., \& Seydell, A. (2008). Visual attention amplifies response priming of pointing movements to color targets. Perception \& Psychophysics, 70, 443-455. doi:10.3758/PP.70.3.443

Schmidt, T., \& Vorberg, D. (2006). Criteria for unconscious cognition: Three types of dissociation. Perception \& Psychophysics, 68, 489504. doi:10.3758/BF03193692

Schmidt, F., Weber, A., \& Schmidt, T. (2014). Activation of response force by self-splitting objects: Where are the limits of feedforward Gestalt processing? Journal of Vision, 14(9), 20. doi:10.1167/14.9. 20. 1-16.

Schubert, T., Palazova, M., \& Hutt, A. (2013). The time course of temporal attention effects on nonconscious prime processing. Attention, 
Perception, \& Psychophysics, 75, 1667-1686. doi:10.3758/s13414013-0515-0

Schwarz, W., \& Mecklinger, A. (1995). Relationship between flanker identifiability and compatibility effect. Perception \& Psychophysics, 57, 1045-1052. doi:10.3758/BF03205463

Sillito, A. M., Cudeiro, J., \& Jones, H. E. (2006). Always returning: Feedback and sensory processing in visual cortex and thalamus. Trends in Neurosciences, 29, 307-316.

Song, J.-H., \& Nakayama, K. (2009). Hidden cognitive states revealed in choice reaching tasks. Trends in Cognitive Sciences, 13, 360-366. doi:10.1016/j.tics.2009.04.009

Sumner, P. (2007). Negative and positive masked-priming - Implications for motor inhibition. Advances in Cognitive Psychology, 3, 317 326. doi:10.2478/v10053-008-0033-0

Sumner, P. (2008). Mask-induced priming and the negative compatibility effect. Experimental Psychology, 55, 133-141.

Sumner, P., Tsai, P.-C., Yu, K., \& Nachev, P. (2006). Attentional modulation of sensorimotor processes in the absence of perceptual awareness. Proceedings of the National Academy of Sciences, 103, 10520-10525.

Thorpe, S., Fize, D., \& Marlot, C. (1996). Speed of processing in the human visual system. Nature, 381, 520-522. doi:10.1038/381520a0

Ulrich, R., \& Miller, J. (2001). Using the jackknife-based scoring method for measuring LRP onset effects in factorial designs. Psychophysiology, 38, 816-827.

van Gaal, S., Ridderinkhof, K. R., van den Wildenberg, W. P. M., \& Lamme, V. A. F. (2009). Dissociating consciousness from inhibitory control: Evidence for unconsciously triggered response inhibition in the stop-signal task. Journal of Experimental Psychology: Human Perception and Performance, 35, 1129-1139. doi:10.1037/ a0013551
VanRullen, R., \& Koch, C. (2003). Visual selective behaviour can be triggered by a feed-forward process. Journal of Cognitive Neuroscience, 15, 209-217.

VanRullen, R., \& Thorpe, S. J. (2001). Is it a bird? Is it a plane? Ultrarapid visual categorisation of natural and artifactual objects. Perception, 30, 655-668. doi:10.1068/p3029

VanRullen, R., \& Thorpe, S. J. (2002). Surfing a spike wave down the ventral stream. Vision Research, 42, 2593-2615.

Vath, N., \& Schmidt, T. (2007). Tracing sequential waves of rapid visuomotor activation in lateralized readiness potentials. Neuroscience, 145, 197-208. doi:10.1016/j.neuroscience.2006.11. 044

Velleman, P. F. (1980). Definition and comparison of robust nonlinear data smoothing algorithms. Journal of the American Statistical Association, 75, 609-615.

Verleger, R., Jaśkowski, P., Aydemir, A., van der Lubbe, R. H. J., \& Groen, M. (2004). Qualitative differences between conscious and nonconscious processing? On inverse priming induced by masked arrows. Journal of Experimental Psychology: General, 133, 494 515. doi:10.1037/0096-3445.133.4.494

Vorberg, D. (2005, April). Ist Hemmung oder Bahnung die Grundlage des umgekehrten Priming-Effekts? Paper presented at the 47th Meeting of Experimentally Working Psychologists, Regensburg, Germany.

Vorberg, D., Mattler, U., Heinecke, A., Schmidt, T., \& Schwarzbach, J. (2003). Different time courses for visual perception and action priming. Proceedings of the National Academy of Sciences, 100, 62756280.

Yantis, S., \& Serences, J. T. (2003). Cortical mechanisms of space-based and object-based attentional control. Current Opinion in Neurobiology, 13, 187-193. 